\title{
Characterization of Admissible Marking Sets in Petri Nets with Conflicts and Synchronizations
}

\author{
Ziyue Ma, Zhiwu Li, and Alessandro Giua
}

August, 2017

\begin{abstract}
In this paper we study the problem of constraint transformation for Petri nets with uncontrollable transitions and containing both conflicts and synchronizations. We show that given an arbitrary net and a set of legal markings, the admissible marking set cannot always be represented by a finite number of disjunctions of GMECs. Moreover, we characterize the GMEC inflation phenomenon, that is, the case in which the representation of the admissible marking set may be too complex to be efficiently implemented in a closed-loop net. To rule out the possibility of GMEC inflation we consider a subclass of constraints called singular GMECs with an acyclic backward-conflict-free uncontrollable subnet. By these assumptions we propose an algorithm to transform a given singular GMEC into a controllable OR-GMEC which precisely characterizes its admissible marking set.
\end{abstract}

\section{Published as:}

[Z.Y. Ma, Z.W. Li, A. Giua, "Characterization of Admissible Marking Sets in Petri Nets with Conflicts and Synchronizations," IEEE Transactions on Automatic Control, Vol. 62, No. 3, pp. 1329-1341, 2017.]

DOI: $10.1109 /$ TAC.2016.2585647.

Ziyue Ma is with School of Electro-Mechanical Engineering, Xidian University, Xi' an 710071, China, and also with Dipartimento di Ingegneria Elettrica ed Elettronica, Università degli Studi di Cagliari, Cagliari, Italy (email: maziyue@gmail.com).

Zhiwu Li is with Institute of Systems Engineering, Macau University of Science and Technology, Taipa, Macau (email: zhwli@xidian.edu.cn, systemscontrol@gmail.com).

Alessandro Giua is with Aix Marseille Université, CNRS, ENSAM, Université de Toulon, LSIS UMR 7296, Marseille 13397, France (email: alessandro.giua@1sis.org) and DIEE, University of Cagliari, Cagliari 09124, Italy (email: giua@diee.unica.it).

This work was supported in part by the National Natural Science Foundation of China under Grant Nos. 61374068, 61472295, the Recruitment Program of Global Experts, and the Science Technology Development Fund, MSAR, under Grant No. 078/2015/A3. 


\section{Introduction}

Supervisory Control Theory (SCT), originated by Ramadge and Wonham [1], provides a unifying framework for modeling and control of discrete event systems (DESs) and has been widely used in modeling various physical systems. In the original work of Ramadge and Wonham [2] automata are used to model plants and specifications, and in recent years Petri nets have been proposed as SCT models since they provide an efficient solution to control problems (e.g., deadlock prevention [3, 4, 5, 6, 7, 8], fault diagnosis [9, 10, 11], identification [12], and marking avoidance $[13,14,15,16,17,18]$ ) without requiring the enumeration of the state space.

In SCT, two types of control requirements, which are called state specifications and language specifications, are usually considered. In [2] Ramadge and Wonham show that a language specification can be converted into a state specification in an extended model obtained by the concurrent composition of the plant and the language specification. As a result, enforcing an arbitrary state specification is a fundamental SCT problem. In Petri nets, a state specification consists in a set of legal states, i.e., markings, and the control objective consists in preventing the system from reaching the illegal states which are also called forbidden markings. Due to the existence of uncontrollable transitions, an illegal marking can be reached from a legal marking by firing uncontrollable transitions. Hence one needs to find a more restrictive control policy which enforces a subset of legal markings, called admissible markings, from which the system cannot reach a forbidden marking by firing uncontrollable transitions.

Both on-line and off-line approaches have been proposed to solve this problem. For live bounded marked graphs, Ghaffari et al. propose an efficient online control policy that is based on the determination of the maximal uncontrollably reachable marking of critical places [19]. For backward-conflict-free-choice nets, by the method of Basile et al., an on-line feedback control logic can be obtained by decomposing the net into a number of marked graph components, which has only polynomial complexity [20]. However, both methods apply to restricted subclasses of nets and the online supervisor must solve a linear programming problem at each step.

On the other hand, off-line approaches have the advantage of providing a closed form solution and do not require complex on-line computations. Many techniques for Petri net marking specifications have been proposed based on reachability analysis. In these approaches, a reachability graph is first computed, and then a suitable control law is established by using the theory of regions [21]. For example, in the method of Uzam and Zhou [22, 23] first-met bad markings (FBMs) are computed from a reachability graph and then are singled out by iteratively adding monitor places. This approach is further improved by Chen [24] to obtain a maximally permissive controller. These approaches work well in systems with a state space of medium size, but for large scale systems they are not applicable due to the state explosion problem. 
To circumvent the need of computing a full reachability graph, a different off-line approach is based on the notion of constraint transformation: it seeks a solution by directly characterizing the set of admissible markings that corresponds to a given legal marking set, without enumerating the reachability space. Generalized Mutual Exclusion Constraints (GMECs) [25] are a common type of linear constraints which defines a set of legal markings. A GMEC can be easily enforced by a control structure composed of a single monitor place [25], while OR-AND GMECs that are a disjunction of conjunctive GMECs can be enforced by a monitor-switcher control structure [17].

A GMEC transformation substitutes a given uncontrollable GMEC by one or more controllable GMECs whose legal set is contained in the admissible marking set of the original constraint. Moody and Antsaklis proposed a method to transform a given uncontrollable GMEC into a new controllable one [14]. Their approach is very efficient from a computational point of view but the solution is not guaranteed to be maximally permissive, i.e., it may restrict the plant within a strict subset of the admissible marking set. Holloway et $a l$. studied a very similar problem and proposed an algorithm to estimate the maximal number of tokens that a place may uncontrollably receive at a given marking [26]. Luo et al. and Wang et al. proposed several GMEC transformation algorithms that have been shown to be maximally permissive for different subclasses of Petri nets [27, 28, 29]. However, these methods are restrictive since they can only be applied to ordinary and synchronization-free nets (i.e., each transition has at most one input place). Moreover, we note that there are also some approaches that combine reachability analysis and transformation in both centralized [30] and decentralized cases [31, 32].

In this work, we study the problem of GMEC transformation in Petri nets in a more general setting. The main contributions of this work are summarized as follows.

First, we show that given an arbitrary net containing both conflicts and synchronizations and a set of legal markings $\mathcal{L}_{W_{0}}$ defined by an OR-AND GMEC $W_{0}$, the corresponding admissible marking set $\mathcal{A}_{W_{0}}$ may not be a finite union of integer convex sets and hence cannot be defined by an OR-AND GMEC. However, in the special case in which the set of legal markings is represented by a nonnegative OR-AND GMEC $W_{0}$, the admissible marking set $\mathcal{A}_{W_{0}}$ can always be characterized by a transformed OR-AND GMEC $W$. Nevertheless, besides the nonnegligible difficulty in finding such a transformed constraint, $W$ may contain the disjunction of a very large number of GMECs. This number depends not only on the constraint and the structure of the net but also on the bound of the constraint. We call this phenomenon the GMEC inflation.

To rule out the undesirable phenomenon of the GMEC inflation, we consider two assumptions. The first assumption requires that the legal marking set to be enforced be characterized by a particular type of GMECs called singular GMECs which are fairly general and of practical usage. The second assumption requires that the influenced uncontrollable subnet (defined in Section IV) of the singular GMEC be backward-conflictfree (BCF) and acyclic. A BCF net is an ordinary net in which each place has at most one input transition: 
this class of nets can model both conflicts (e.g., two or more workflows share the same source buffer) and synchronizations (e.g., two or more products are assembled) in the workflow systems. We show that these two assumptions are sufficient to exclude the possibility of the GMEC inflation.

Finally, for a singular GMEC whose influenced uncontrollable subnet is acyclic and BCF, we propose a method to transform it into a controllable OR-GMEC which precisely represents its admissible marking set. This transformation is based on the expansion and composition of singular GMECs. To the best of our knowledge, no other method has been presented in the literature for maximally permissive GMEC transformation in the nets containing both conflicts and synchronization. Such a resulting OR-GMEC can be further implemented by a Petri net controller [15, 17, 33].

The paper is organized in seven sections. Section II recalls the basic notions of Petri nets and GMECs. Section III studies the properties of admissible marking sets and the GMEC inflation. Section IV introduces the notion of singularity of GMECs, GMEC expansion and compositions, and their properties are explored. In Section $\mathrm{V}$ an algorithm based on the GMEC composition operation is proposed to transform a given singular GMEC into a controllable OR-GMEC that is maximally permissive, and the correctness of the algorithm is proved in Section VI. Section VII draws the conclusions.

\section{Preliminaries}

\subsection{Petri Net}

A Petri net is a four-tuple $N=(P, T$, Pre, Post $)$, where $P$ is a set of $m$ places represented by circles; $T$ is a set of $n$ transitions represented by bars; Pre : $P \times T \rightarrow \mathbb{N}$ and Post $: P \times T \rightarrow \mathbb{N}$ are the pre- and post-incidence functions that specify the arcs in the net and are represented as matrices in $\mathbb{N}^{m \times n}$ (here $\mathbb{N}=\{0,1,2, \ldots\}$ ). The incidence matrix of a net is defined by $C=$ Post - Pre $\in \mathbb{Z}^{m \times n}$ (here $\mathbb{Z}=\{0, \pm 1, \pm 2, \ldots\})$. A net is ordinary if $\operatorname{Pre}(p, t), \operatorname{Post}(p, t) \in\{0,1\}$ for all $p \in P$ and $t \in T$.

For a transition $t \in T$ we define its set of input places as ${ }^{\bullet} t=\{p \in P \mid \operatorname{Pre}(p, t)>0\}$ and its set of output places as $t^{\bullet}=\{p \in P \mid \operatorname{Post}(p, t)>0\}$. The notions for ${ }^{\bullet} p$ and $p^{\bullet}$ are analogously defined.

A marking is a vector $M: P \rightarrow \mathbb{N}$ that assigns to each place of a Petri net a non-negative integer number of tokens, represented by black dots. A marking can also be represented as an $m$-component vector. We denote by $M(p)$ the marking of place $p$. A marked net $\left\langle N, M_{0}\right\rangle$ is a net $N$ with an initial marking $M_{0}$.

A transition $t$ is enabled at $M$ if $M \geq \operatorname{Pre}(\cdot, t)$ and may fire reaching a new marking $M^{\prime}=M_{0}+C(\cdot, t)$. We write $M[\sigma\rangle$ to denote that the sequence of transitions $\sigma$ is enabled at $M$, and we write $M[\sigma\rangle M^{\prime}$ to denote that the firing of $\sigma$ yields $M^{\prime}$. We denote by $R\left(N, M_{0}\right)$ the set of all markings reachable from the initial one. 
The transition set $T$ can be partitioned into $T_{c}$ and $T_{u}$ which represent the controllable and uncontrollable transition sets, respectively, i.e., $T=T_{c} \cup T_{u}$ and $T_{c} \cap T_{u}=\emptyset$. A transition $t_{u} \in T_{u}$ is not controllable, i.e., it cannot be disabled by a control agent (e.g., control places).

Given a net $N=(P, T$, Pre, Post $), \hat{N}=(\hat{P}, \hat{T}, \hat{P}$ re,$\hat{P}$ ost $)$ is called a subnet of $N$ if $\hat{P} \subset P, \hat{T} \subset T$ and $\hat{P}$ re (resp., $\hat{P}_{\text {ost }}$ ) is the restriction of Pre (resp., Post) to $\hat{P} \times \hat{T}$.

In a net $N=(P, T$, Pre, Post $)$, a path is a sequence of nodes $\pi=x_{1} x_{2} \cdots x_{k}$ such that $x_{i} \in P \cup T$ for all $i=1, \ldots, k$, and $x_{i} \in{ }^{\bullet} x_{i+1}$ for all $i=1, \ldots, k-1$. A node $x_{1} \in P \cup T$ is said to be in the downstream of $x_{2} \in P \cup T$ if there exists a path from $x_{2}$ to $x_{1}$. The set of nodes in the upstream (resp., downstream) of a node $x$ is denoted as $(\bullet x)^{\infty}$ (resp., $\left(x^{\bullet}\right)^{\infty}$ ).

\subsection{GMEC}

A Generalized Mutual Exclusion Constraint (GMEC) is a pair $(\mathbf{w}, k)$ where $\mathbf{w} \in \mathbb{Z}^{m}$ and $k \in \mathbb{N}$. A GMEC defines a set of legal markings:

$$
\mathcal{L}_{(\mathbf{w}, k)}=\left\{M \in \mathbb{N}^{m} \mid \mathbf{w}^{T} \cdot M \leq k\right\}
$$

The objective of a supervisor is to ensure that only legal markings are reached by preventing transition firings that yield forbidden markings in the set $\mathcal{F}=\mathbb{N}^{m} \backslash \mathcal{L}_{(\mathbf{w}, k)}$. However, the presence of uncontrollable transitions may complicate the problem. In fact, given a net $N$ with set of uncontrollable transitions $T_{u}$, it may occur that from a legal marking $M \in \mathcal{L}_{(\mathbf{w}, k)}$ a sequence of uncontrollable transitions, which cannot be disabled by the supervisor, yields a forbidden marking. For this reason the supervisor needs to restrict the evolution of the system within the set of admissible markings denoted by $\mathcal{A}_{(\mathbf{w}, k)}$ :

$$
\mathcal{A}_{(\mathbf{w}, k)}=\left\{M \in \mathbb{N}^{m} \mid \forall \sigma_{u} \in T_{u}^{*}, M\left[\sigma_{u}\right\rangle M^{\prime} \in \mathcal{L}_{(\mathbf{w}, k)}\right\}
$$

where $T_{u}$ is the set of uncontrollable transitions of the given net $N$.

An AND-GMEC [17] is a pair $(\mathbf{W}, \mathbf{k})$ where $\mathbf{W} \in \mathbb{Z}^{s \times m}$ and $\mathbf{k} \in \mathbb{N}^{s}$. An AND-GMEC defines a set of legal markings:

$$
\mathcal{L}_{(\mathbf{W}, \mathbf{k})}=\left\{M \in \mathbb{N}^{m} \mid \mathbf{W}^{T} \cdot M \leq \mathbf{k}\right\}
$$

and a set of admissible markings:

$$
\mathcal{A}_{(\mathbf{W}, \mathbf{k})}=\left\{M \in \mathbb{N}^{m} \mid \forall \sigma_{u} \in T_{u}^{*}, M\left[\sigma_{u}\right\rangle M^{\prime} \in \mathcal{L}_{(\mathbf{W}, \mathbf{k})}\right\}
$$




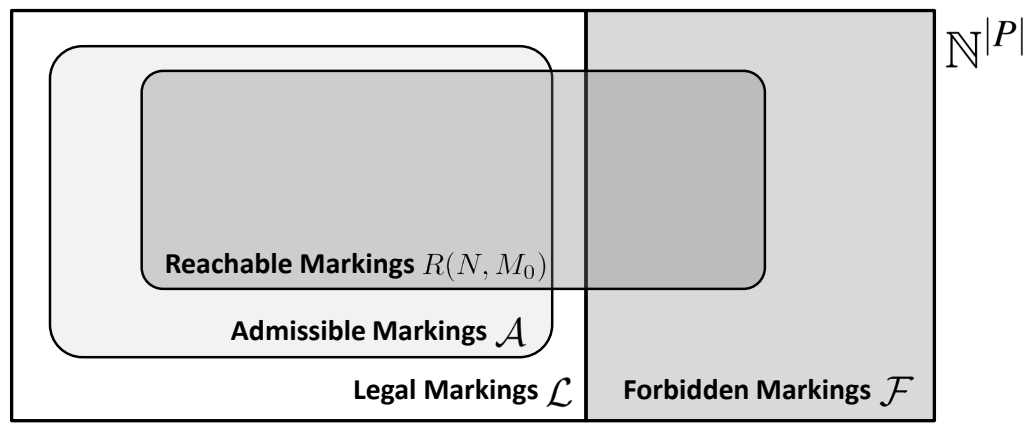

Figure 1: The graphic illustration of legal, admissible, forbidden, and reachable marking sets.

An OR-AND GMEC [17] is a set: $W=\left\{\left(\mathbf{W}_{1}, \mathbf{k}_{1}\right), \ldots,\left(\mathbf{W}_{r}, \mathbf{k}_{r}\right)\right\}$ where $\left(\mathbf{W}_{i}, \mathbf{k}_{i}\right)$ is an AND-GMEC for $i \in\{1, \ldots, r\}$. An OR-GMEC defines a set of legal markings:

$$
\mathcal{L}_{W}=\left\{M \in \mathbb{N}^{m} \mid \exists\left(\mathbf{W}_{i}, \mathbf{k}_{i}\right) \in W, \mathbf{W}_{i}^{T} \cdot M \leq \mathbf{k}_{i}\right\}
$$

and a set of admissible markings:

$$
\mathcal{A}_{W}=\left\{M \in \mathbb{N}^{m} \mid \forall \sigma_{u} \in T_{u}^{*}, M\left[\sigma_{u}\right\rangle M^{\prime} \in \mathcal{L}_{W}\right\}
$$

Definition 1 (Controllability of GMECs) [34] Given a net $N$ with set of uncontrollable transitions $T_{u}$ and incidence matrix $C$, a single GMEC $(\boldsymbol{w}, k)$ is said to be structurally controllable if $\forall t \in T_{u}, \boldsymbol{w}^{T} \cdot C(\cdot, t) \leq 0$ holds. An OR-AND GMEC is said to be structurally controllable if all single GMECs in it are structurally controllable.

Proposition 1 If $(\boldsymbol{w}, k)$ is controllable, then $\mathcal{L}_{(w, k)}=\mathcal{A}_{(w, k)}$ holds.

Proof: First, it is obvious that $\mathcal{L}_{(\mathbf{w}, k)} \supseteq \mathcal{A}_{(\mathbf{w}, k)}$ holds. Second, For any marking $M \in \mathcal{L}_{(\mathbf{w}, k)}$ and any $\sigma \in T_{u}^{*}$ such that $M\left[\sigma_{u}\right\rangle M^{\prime}, \mathbf{w}^{T} \cdot M^{\prime}=\mathbf{w}^{T} \cdot\left(M+C \cdot \mathbf{y}_{\sigma}\right) \leq \mathbf{w}^{T} \cdot M$, since $\mathbf{w}^{T} \cdot C(\cdot, t) \leq 0$ holds for all $t \in T_{u}$. This indicates that $M^{\prime} \in \mathcal{L}_{(\mathbf{w}, k)}$ and thus $M \in \mathcal{A}_{(\mathbf{w}, k)}$ holds, i.e., $\mathcal{L}_{(\mathbf{w}, k)} \subseteq \mathcal{A}_{(\mathbf{w}, k)}$ holds, which concludes the proof.

For simplicity, in the sequel we call a GMEC "controllable" if it is structurally controllable. By Proposition 1, if a GMEC is controllable, then the firing of any uncontrollable sequence at any legal marking does not reach a marking that is illegal. A controllable GMEC can be enforced on a marked net $\left\langle N, M_{0}\right\rangle$ by adding a monitor place $p_{s}$ with $C\left(p_{s}, t\right)=-\mathbf{w}^{T} \cdot C(\cdot, t)$ and $M_{0}\left(p_{s}\right)=k-\mathbf{w}^{T} \cdot M_{0}$ (an analogous control mechanism is also used to design a controller for OR-AND GMECs [17]).

In general, an uncontrollable GMEC $(\mathbf{w}, k)$ cannot be enforced since the monitor place may attempt to 
disable some uncontrollable transitions. Note, however, that for some particular initial marking $M_{0}$ it may happen that the monitor place corresponding to an uncontrollable GMEC does not disable uncontrollable transitions at any reachable marking ${ }^{1}$. However, there is no efficient way to determine this except checking the entire reachability graph. Furthermore, this property depends on the intial marking $M_{0}$. On the contrary, the controllability of a GMEC can be easily verified by incidence matrix analysis [35] and provides a feasible solution for all initial markings.

\subsection{Integer convex sets}

A set $X \subseteq \mathbb{R}^{m}$ (R is the set of real numbers) is convex if $\left(x_{1}, x_{2} \in X\right) \Rightarrow\left((\forall \lambda \in[0,1]) \lambda \cdot x_{1}+(1-\lambda) \cdot x_{2} \in\right.$ $X)$.

A set $S \subseteq \mathbb{N}^{m}$ is said to be an integer convex set if there exists a convex set $S_{\text {real }} \subseteq \mathbb{R}^{m}$ such that $S=S_{\text {real }} \cap \mathbb{N}^{m}$.

According to the previous definition, an integer convex set $S$ is the set of integer points contained in a corresponding real convex set $S_{\text {real }}$. For example, the set $S=\left\{x_{1}, x_{2}, x_{3}\right\}$ where $x_{1}=[0,0]^{T}, x_{2}=[0,1]^{T}$, and $x_{3}=[1,0]^{T}$, is an integer convex set since there exists $S_{\text {real }}=\{(a, b) \in \mathbb{R} \times \mathbb{R} \mid(a \geq 0) \wedge(b \geq$ $0) \wedge(a+b \leq 1)\}$ such that $S=S_{\text {real }} \cap \mathbb{N}^{m}$.

Note that given two integer convex sets $S_{1}$ and $S_{2}, S=S_{1} \cap S_{2}$ is also an integer convex set since $S=S_{1} \cap S_{2}=\left(S_{1, \text { real }} \cap \mathbb{N}^{m}\right) \cap\left(S_{2, \text { real }} \cap \mathbb{N}^{m}\right)=\left(S_{1, \text { real }} \cap S_{2, \text { real }}\right) \cap \mathbb{N}^{m}$ where $S_{1, \text { real }} \cap S_{2, \text { real }}$ is a real convex set.

From the definitions of GMECs above, two properties trivially hold.

Property 1 The legal marking set defined by an AND-GMEC (i.e., $\mathcal{L}_{(\boldsymbol{W}, \boldsymbol{k})}$ ) is an integer convex set.

Property 2 The legal set defined by an OR-AND GMEC (i.e., $\mathcal{L}_{W}$ ) is a finite union of integer convex sets.

\section{GMEC Characterization of Admissible Marking Sets}

Assume that the legal marking set to be enforced on a Petri net is defined by an OR-AND GMEC $W_{0}$. Due to the presence of uncontrollable transitions, such a constraint may be uncontrollable and hence cannot be directly enforced, as discussed at the end of Section II.B.

\footnotetext{
${ }^{1}$ Such GMECs are called admissible GMECs in the literature [14]. We note that the admissibility of a GMEC depends on the initial marking $M_{0}$, while the set of admissible markings does not. Obviously a controllable GMEC is always admissible regardless of the initial marking, but the converse is not true.
} 
To solve this problem, one may determine a different OR-AND GMEC $W$ to meet the control demand. The new constraint must satisfy two conditions: (1) $W$ must be controllable, i.e., $\mathcal{L}_{W}=\mathcal{A}_{W}$ (otherwise it cannot be enforced); (2) the legal marking set defined by $W$ must be a subset of $\mathcal{A}_{W_{0}}$, i.e., $\mathcal{L}_{W} \subseteq \mathcal{A}_{W_{0}}$. The set of OR-AND GMECs that satisfy the two conditions are denoted as $\Omega\left(W_{0}\right)$, i.e., $\Omega\left(W_{0}\right)=\left\{W \mid\left(\mathcal{L}_{W} \subseteq\right.\right.$ $\left.\mathcal{A}_{W_{0}}\right) \wedge(W$ is controllable $\left.)\right\}$.

Among all OR-AND GMECs in $\Omega\left(W_{0}\right)$, we look for constrains whose set of legal marking is maximal, so as to minimally restrict the behavior of the controlled net. This motivates the following definition that identifies the "optimal" solution to the considered control problem. ${ }^{2}$

Definition 2 Given a Petri net $N$ and an OR-AND GMEC $W_{0}$, an OR-AND GMEC $W \in \Omega\left(W_{0}\right)$ is said to be maximally permissive (with respect to $N$ and $W_{0}$ ) if $\mathcal{L}_{W}=\mathcal{A}_{W_{0}}$.

Now the problem studied in this work is formalized as the following.

Problem 1 Given a net $N=(P, T$, Pre, Post $)$ with $T=T_{c} \cup T_{u}$ and an OR-AND GMEC $W_{0}$, determine a maximally permissive OR-AND GMEC $W$.

In the literature, GMEC transformation techniques are used to solve Problem 1 for subclasses of nets. In the approaches based on transformation, uncontrollable GMECs are iteratively substituted by new ones until a maximally permissive solution is reached [14, 26, 27, 28, 29]. However, in the following we show that this scenario is not possible for arbitrary nets and initial GMEC $W_{0}$, since in some cases $\mathcal{A}_{W_{0}}$ cannot be characterized as the legal set of an OR-AND GMEC. In other cases, such a characterization is possible but requires such a large number of disjunctive constraints that is practically useless.

\subsection{Problem 1 with Arbitrary Nets and GMECs}

We first present a negative result, i.e., we show that given an arbitrary net and an arbitrary OR-AND GMEC $W_{0}$, there does not always exist a maximally permissive OR-AND GMEC $W$. We first present the following lemma.

Lemma 1 Given a Petri net $N$ containing $m$ places, let $W$ be an OR-AND GMEC with set of legal markings $\mathcal{L}_{W}$. For any integer convex set $X \subseteq \mathbb{N}^{m}$, the intersection $\mathcal{L}_{W} \cap X$ is a finite union of integer convex sets.

Proof: From the definition of OR-AND GMECs and Property 2, $\mathcal{L}_{W}$ can be written as $\mathcal{L}_{W}=\bigcup_{i=1}^{r} \mathcal{L}_{i}$ and each $\mathcal{L}_{i}$ is an integer convex set. For any given integer convex set $X \subseteq \mathbb{N}^{m}$, each $\mathcal{L}_{i} \cap X$ is also an

\footnotetext{
${ }^{2}$ In [34] a similar notion called the supremal controllable subset is also discussed, which is defined on the reachability set of a marked net $R\left(N, M_{0}\right)$.
} 


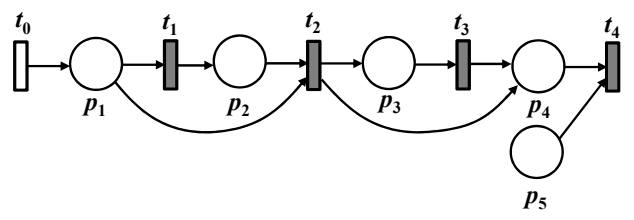

Figure 2: A Petri net for Proposition 2 with $T_{c}=\left\{t_{0}\right\}$ and $T_{u}=\left\{t_{1}, t_{2}, t_{3}, t_{4}\right\}$.

integer convex set. Hence $\mathcal{L}_{W} \cap X=\bigcup_{i=1}^{r}\left(\mathcal{L}_{i} \cap X\right)$ is a finite union of integer convex sets.

By Lemma 1, given a set of markings $S \in \mathbb{N}^{m}$, if we can find an integer convex set $X$ such that $S \cap X$ is not a finite union of integer convex sets (which implies that both $S$ and $X$ are infinite sets), then there does not exist an OR-AND GMEC $W$ such that $\mathcal{L}_{W}=S$.

Proposition 2 [Non-Existence of a GMEC Solution] There exist some Petri nets and OR-AND GMECs whose admissible marking set cannot be characterized by an OR-AND GMEC.

Proof: We prove this by the following counterexample. In Figure 2, let $T_{c}=\left\{t_{0}\right\}$ and $T_{u}=$ $\left\{t_{1}, t_{2}, t_{3}, t_{4}\right\}$. Consider an OR-AND GMEC $W_{0}$ consisting of a single GMEC $\left(\mathbf{w}_{0}, k_{0}\right) \equiv M\left(p_{4}\right)-$ $M\left(p_{5}\right) \leq 0$. The admissible marking set $\mathcal{A}_{W_{0}} \subset \mathbb{N}^{5}$ is too complicated to be defined explicitly. Consider the marking set $X=\left\{M \in \mathbb{N}^{5} \mid M\left(p_{2}\right)=M\left(p_{3}\right)=M\left(p_{4}\right)=0\right\}$ which is a convex subset of $\mathbb{N}^{5}$. The set $\mathcal{A}_{W_{0}} \cap X$ contains the points in the shaded area in Figure 3 including the boundary.

One can readily find that $\mathcal{A}_{W_{0}} \cap X$ cannot be represented by an OR-AND GMEC. Actually the following infinite union of AND-GMECs characterizes $\mathcal{A}_{W_{0}} \cap X$ :

$$
\left.\bigvee_{j=0}^{+\infty}\left(M\left(p_{1}\right) \leq 2 j+1\right) \wedge\left(M\left(p_{5}\right) \geq 2 j\right)\right)
$$

As a result, $\mathcal{A}_{W_{0}} \cap X$ cannot be written as a finite union of integer convex sets. By Lemma $1, \mathcal{A}_{W_{0}}$ is not a the legal set of an OR-AND GMEC.

\subsection{Problem 1 with Nonnegative GMECs and GMEC Inflation}

It has been proved $[36,37]$ that if the uncontrollable subnet is ordinary and synchronization-free (i.e., each transition has at most one input place), and if the initial legal marking is characterized by a single nonnegative $\left(\mathbf{w}_{0}, k_{0}\right)$, then the admissible marking set of $\left(\mathbf{w}_{0}, k_{0}\right)$, i.e., $\mathcal{A}_{\left(\mathbf{w}_{0}, k_{0}\right)}$, can always be characterized by an AND-GMEC. Here we show a generalized result stating that given a nonnegative OR-AND GMEC $W_{0}$, its 


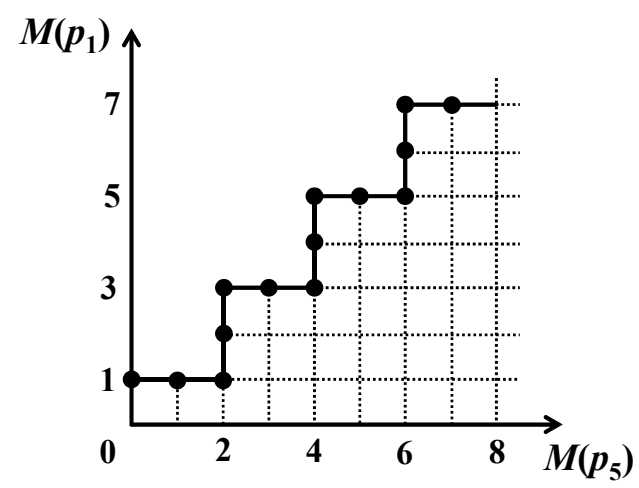

Figure 3: The admissible marking set of the net in Figure 2 with the uncontrollable GMEC $M\left(p_{4}\right)-M\left(p_{5}\right) \leq$ 0 .

admissible marking set can always be characterized by an OR-AND GMEC $W$ regardless the structure of the net.

Definition 3 An AND-GMEC $(\boldsymbol{W}, \boldsymbol{k})$ is said to be nonnegative if $\boldsymbol{W} \geq \boldsymbol{0}$. An OR-AND GMEC is said to be nonnegative if all AND-GMECs in it are nonnegative.

Given an OR-AND GMEC $W_{0}$, if $\forall\left(\mathbf{W}_{i}, \mathbf{k}_{i}\right) \in W_{0}, \mathbf{W}_{i}$ is nonnegative, then there always exists an OR-AND GMEC $W$ such that $\mathcal{L}_{W}=\mathcal{A}_{W_{0}}$. To prove this we first recall the well-known Dickson's Lemma.

Lemma 2 [Dickson's Lemma][38] Let $S \subseteq \mathbb{N}^{m}$ be a right-closed set ${ }^{3}$. Then the set $S_{\min }$ of minimal elements of $S$ for the ordering $\leq$ is finite.

Proposition 3 Given a Petri net $N=\left(P, T\right.$, Pre, Post) in which $T=T_{c} \cup T_{u}$ and an OR-AND GMEC $W_{0}=\left\{\left(\boldsymbol{W}_{1}, \boldsymbol{k}_{1}\right), \ldots,\left(\boldsymbol{W}_{r}, \boldsymbol{k}_{r}\right)\right\}$ such that $\boldsymbol{W}_{i} \geq \boldsymbol{0}$ for $i=1, \ldots, r$, there exists an OR-AND GMEC W such that $\mathcal{L}_{W}=\mathcal{A}_{W_{0}}$.

Proof: Consider an arbitrary $\left(\mathbf{W}_{i}, \mathbf{k}_{i}\right) \in W_{0}$. Let $\mathcal{F}_{i}=\complement \mathcal{A}_{\left(\mathbf{W}_{i}, \mathbf{k}_{i}\right)}$, i.e., the complement set of $\mathcal{A}_{\left(\mathbf{W}_{i}, \mathbf{k}_{i}\right)}$. Since for any marking $M \in \mathcal{F}_{i}$, there exists a firing sequence $\sigma_{u} \in T_{u}^{*}$ such that $M\left[\sigma_{u}\right\rangle M^{\prime} \notin \mathcal{L}_{\left(\mathbf{W}_{i}, \mathbf{k}_{i}\right)}$. Then for any $\hat{M} \geq M \in \mathcal{F}_{i}, \hat{M}\left[\sigma_{u}\right\rangle \hat{M}^{\prime} \geq M^{\prime}$ where $\mathbf{W}_{i}^{T} \cdot \hat{M}^{\prime} \not \mathbf{k}$ holds due to $\mathbf{W}_{i} \geq \mathbf{0}$, which indicates the truth of $\hat{M} \notin \mathcal{L}_{\left(\mathbf{W}_{i}, \mathbf{k}_{i}\right)}$. Hence $\mathcal{F}_{i}$ is right-closed. According to Dickson's Lemma, the set composed by the minimal elements of $\mathcal{F}_{i}$, denoted as $\mathcal{F}_{\text {min, } i}$, is finite. As a result, $\mathcal{F}_{i}$ can be represented as a finite union of integer convex sets:

$$
\mathcal{F}_{i}=\bigcup_{M^{\prime} \in \mathcal{F}_{m i n, i}}\left\{M \in \mathbb{N}^{m} \mid M \geq M^{\prime}\right\}
$$

\footnotetext{
${ }^{3}$ A set $S \subseteq \mathbb{N}^{m}$ is said to be right-closed if $(x \in S, y \geq x) \Rightarrow(y \in S)$.
} 
which can be enforced by a disjunction of conjunctive inequalities denoted as $\mathcal{I}_{i}$. Consequently, $\mathcal{A}_{W_{0}}$ can be written as:

$$
\mathcal{A}_{W_{0}}=\complement\left(\bigcap_{i=1}^{r} \mathcal{F}_{i}\right)
$$

and $\mathcal{A}_{W_{0}}$ can be characterized by a logical expression $\mathcal{I}=\neg \bigwedge_{i=1}^{r} \mathcal{I}_{i}$ consisting of linear inequalities. The disjunctive normal form of $\mathcal{I}$ is a finite union of integer convex sets and hence, by Property 2 , can be enforced by an OR-AND GMEC.

Although Proposition 3 ensures the existence of an OR-AND GMEC $W$ such that $\mathcal{L}_{W}=\mathcal{A}_{W_{0}}$ in case that $W_{0}$ is nonnegative, it cannot be used to determine the OR-AND GMEC $W$, since it is difficult to compute the minimal elements of $\mathcal{F}_{i}$ for each AND-GMEC. In general an exhaustive enumeration of markings in the space $\mathbb{N}^{m}$ is needed, which is even worse than enumerating the reachability space. Moreover, even if it is possible to find the expected OR-AND GMEC $W$, it can be too complex to be practically implemented as a closed-loop net, as shown in the following example.

Example 1 Consider the Petri net in Figure 4(a). The initial inadmissible GMEC $W_{0}$ consists of a single GMEC $\left(\boldsymbol{w}_{0}, k_{0}\right) \equiv M\left(p_{4}\right) \leq k_{0}$. By applying Lemma 1 we consider the set $\mathcal{A}_{W_{0}} \cap X$ where $X=\{M \in$ $\left.\mathbb{N}^{4} \mid M\left(p_{2}\right)=M\left(p_{3}\right)=0\right\}$. It is trivial that $\mathcal{A}_{W_{0}} \cap X$ can be characterized by the following finite OR-AND GMEC $W^{\prime}$ :

$$
\left.\bigvee_{j=0}^{\left\lfloor k_{0} / 2\right\rfloor+1}\left(M\left(p_{1}\right) \leq 2 j+1\right) \wedge\left(M\left(p_{4}\right) \leq k_{0}-2 j\right)\right)
$$

and the admissible marking sets for $k_{0}=3,5$, and 999 are illustrated in Figure 5. As a result, there will be $\left\lfloor k_{0} / 2\right\rfloor+1$ AND-GMECs in $W^{\prime}$, and one can verify that all these AND-GMECs are not redundant. Therefore for $k_{0}=999$, in the original $\mathbb{N}^{4}$ space an OR-AND GMECW satisfying $\mathcal{L}_{W}=\mathcal{A}_{W_{0}}$ contains at least 500 AND-GMECs. To enforce an OR-AND GMEC as a closed-loop supervisor, by using the technique in [17] (which synthesizes a closed-loop controller with the lowest structural complexity) the number of additional transitions (in [17] they are called mirror transitions) is in the order of $O\left(r^{2}\right)$ where $r$ is the number of AND-GMECs. To enforce such an OR-AND GMEC $W$ more than 250,000 (i.e., $\left.\left(\left\lfloor k_{0} / 2\right\rfloor+1\right)^{2}\right)$ additional transitions will be added for a single transition in the plant. As a result, although such an OR-AND GMEC $W$ exists, it is practically infeasible to be used as a controller in real systems.

From Example 1 we observe a phenomenon we call the GMEC inflation. The number of GMECs in the OR-AND GMEC $W$ describing the admissible marking set $\mathcal{A}_{\left(\mathbf{w}_{0}, k_{0}\right)}$ depends not only on the structure of the constraint and of the net but also on the parameter $k_{0}$, i.e., the constraint bound. Since the constraint bound $k_{0}$ is related to the capacity of the physical plant system, the GMEC inflation may occur in real systems with large capacity, even if the net structure is rather simple (e.g., Figure 4(a)). For the net in Figure 4(a) with 


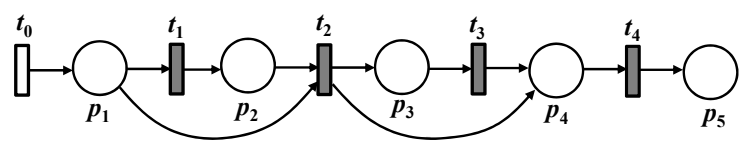

(a)

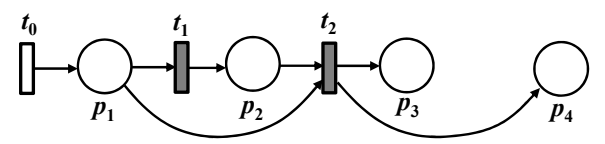

(b)

Figure 4: Two Petri nets for Examples 1 and 4.

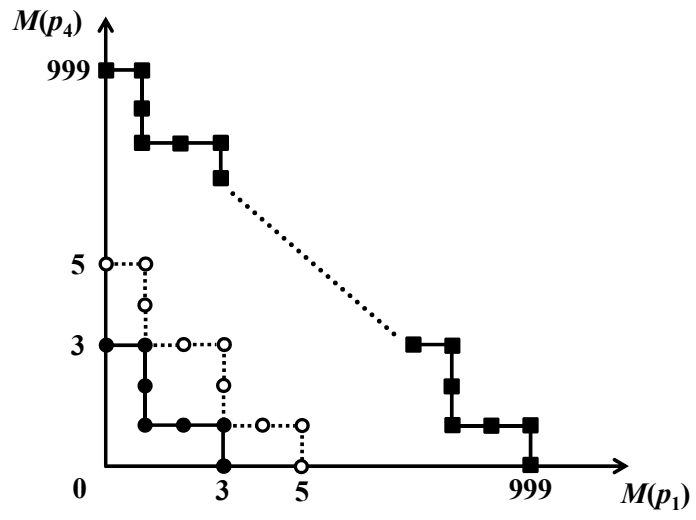

Figure 5: The admissible marking set of the net in Figure 4(a) with the uncontrollable GMEC $M\left(p_{4}\right) \leq k_{0}$. Solid circle border markings $(\bullet)$, empty circle border markings (०), and solid box border markings (ם) and all markings down-left to the borders belong to the admissible marking set $\mathcal{A}_{\left(\mathrm{w}_{0}, k_{0}\right)} \cap X$ for $k_{0}=3,5$, and 999 , respectively. 
$k_{0}=999$, the OR-AND GMEC $W$ that describes the set of admissible markings requires more than 1,500 additional places and 250,000 additional transitions, which indicates that the closed-loop net has an incidence matrix in $\mathbb{Z}^{1,500 \times 250,000}$ that can hardly be further analyzed.

Since the GMEC inflation greatly increases the complexity of the controller, to avoid it one may try to modify the structure of the uncontrollable subnet, e.g., adding additional control logic to make some uncontrollable transitions become controllable. We find that the GMEC inflation does not occur in some restrictive subclasses of Petri nets (e.g., synchronization-free ordinary nets [27] and conflict-free ordinary nets [13]). On the other hand, Example 1 is a net containing both a conflict place and a synchronization transition. Conflict and synchronization structures are very common in resource allocation systems, which motivates us to study the GMEC transformation in Petri nets containing both primitives.

\section{Singularity of GMECs and Problem Statement}

This section introduces a particular type of GMECs called singular GMECs. We show that the GMEC inflation would not occur in case that the initial GMEC is singular and its influenced uncontrollable subnet is backward-conflict-free.

\subsection{Singular GMECs and Backward-conflict-free Nets}

We first define the root place of a given GMEC and then introduce singular GMECs.

Definition 4 Given a net $N$ and a GMEC $(\boldsymbol{w}, k), p_{x} \in|\boldsymbol{w}|$ is called a root place of $|\boldsymbol{w}|$ if $|\boldsymbol{w}| \subseteq\left(p_{x}^{\bullet}\right)^{\infty}$, where $|\boldsymbol{w}|$ denotes the support ${ }^{4}$ of $\boldsymbol{w}$ and $\left(p_{x}^{\bullet}\right)^{\infty}$ denotes the set of all nodes in the downstream of $p$.

In other words, given a GMEC $(\mathbf{w}, k)$, a root place $p_{x}$ is a place that belongs to the support of $\mathbf{w}$ such that all the places in the support of $\mathbf{w}$ are in its downstream. The root place of a GMEC may be unique, multiple, or not exist.

Definition 5 Given a Petri net $N$, a GMEC $(\boldsymbol{w}, k)$ is said to be singular if:

1. its root place $p_{x}$ exists and is unique;

2. there exists at most one transition $t_{x}$ such that $\boldsymbol{w}^{T} \cdot C\left(\cdot, t_{x}\right)>0$;

3. if $t_{x}$ exists, then $t_{x} \cap|\boldsymbol{w}|=\emptyset, t_{x}^{\bullet} \cap|\boldsymbol{w}|=\left\{p_{x}\right\}$, and $\boldsymbol{w}^{T} \cdot C\left(\cdot, t_{x}\right)=1$.

\footnotetext{
${ }^{4}$ In a net $N$ with $m$ places, the support of a vector $\mathbf{w} \in \mathbb{N}^{m}$ is $\{p \in P \mid w(p) \neq 0\}$.
} 
For a singular GMEC $(\boldsymbol{w}, k)$, such a transition $t_{x}$, if it exists, is called its injection transition.

The concept of singular GMECs involves the fact that there exists at most one transition $t_{x}$ whose firing will add one token to the root place $p_{x}$ (whose weight is $w\left(p_{x}\right)=1$ ) but will not remove tokens from any support place of $\mathbf{w}$, while the firing of all other transitions will not increase the token count of $\mathbf{w}$.

Checking if a GMEC is singular is not difficult: to verify Conditions 1 and 3 one could simply check the incidence matrix of the net, and to check Condition 2 one needs to do $\left|T_{u}\right|$ vector multiplications. By definition, a singular GMEC has the following property.

Proposition 4 For any singular GMEC the firing of any transition $t$ will increase its token count by one at most.

Proof: Trivial, since the firing of its injection transition $t_{x}$ (if it exists) increases its token count by one, while the firing of any transition $t \neq t_{x}$ does not increase its token count.

Notice that the injection transition is the only transition whose firing would increase the token count of a singular GMEC, we have the following result.

Corollary 1 A singular GMEC with no injection transition is controllable.

Example 2 Figure 6 illustrates an automated factory equipped with robots and AGVs. Raw material A arrives at Workplace 1 and then is transported by an AGV to Workplace 2. Material A can be assembled with raw material $B$ from either Workplace 3 or Workplace 4 by two different robots, and the product is transported to Workplaces 5 and 6, respectively. Another robot assembles parts from Workplaces 5 and 6 and the product is transported to Storehouse 7.

The Petri net model is depicted in Figure 7 in which $T_{u}=\left\{t_{1}, t_{2}, t_{3}, t_{4}\right\}$. In the net in Figure 7, the GMEC $M\left(p_{2}\right)+M\left(p_{5}\right)+M\left(p_{6}\right)+2 M\left(p_{7}\right) \leq 3$ is singular with root place $p_{2}$ and injection transition $t_{1}$. The GMEC $M\left(p_{1}\right)+M\left(p_{2}\right)+M\left(p_{5}\right)+M\left(p_{7}\right) \leq 1$ is also singular with root place $p_{1}$ but no injection transition. On the contrary, the GMECs $2 M\left(p_{5}\right)+M\left(p_{7}\right) \leq 1$ and $M\left(p_{3}\right)+M\left(p_{5}\right)+2 M\left(p_{7}\right) \leq 3$ are not singular.

Singular GMECs are common as a state specification. For example, in monolithic supervisor trimming problems [16], the initial GMEC to be enforced is usually in the form $k_{1} M(p) \leq k_{2}\left(k_{1}>0\right)$ that can always be converted into an equivalent GMEC $M(p) \leq\left\lfloor k_{2} / k_{1}\right\rfloor$ where $\lfloor x\rfloor$ denotes the maximal integer which does not exceed $x$. Such a GMEC is singular if place $p$ has one single input arc whose weight is 1 . In the sequel we make the first assumption on the initial GMEC $\left(\mathbf{w}_{0}, k_{0}\right)$ to be enforced. 
Assumption 1 (Initial Singularity Assumption) The initial GMEC $\left(\boldsymbol{w}_{0}, k_{0}\right)$ to be enforced is singular.

However, only Assumption 1 is not sufficient to rule out the possibility of the GMEC inflation, as will be shown at the end of this subsection. In the following we make another assumption on the net structure.

Notice that given an uncontrollable GMEC $(\mathbf{w}, k)$ to be enforced, there may exist some places $p \in P$ whose tokens will never uncontrollably flow to the support places of $\mathbf{w}$. Hence practically we only need to consider the subnet which may potentially affect the token count of $(\mathbf{w}, k)$.

Definition 6 The influenced uncontrollable subnet of a $\operatorname{GMEC}(\boldsymbol{w}, k)$ in $N$, denoted as $\hat{N}=(\hat{P}, \hat{T}, \hat{P}$ re,$\hat{P}$ ost $)$, is the subnet of $N$ obtained by removing all transitions $t \in T_{c}$ followed by removing all places $p$ such that $\forall p^{\prime} \in|\boldsymbol{w}|$ there neither exists a path from $p$ to $p^{\prime}$ nor from $p^{\prime}$ to $p$.

Now we make the second assumption on the influenced uncontrollable subnet of the initial GMEC to be enforced.

Definition 7 A backward-conflict-free net (BCF net) is an ordinary Petri net in which each place has at most one input transition, i,e., $\forall p \in P,|\bullet p| \leq 1$ holds.

Assumption 2a (BCF Assumption) The influenced uncontrollable subnet of the initial singular GMEC $\left(\boldsymbol{w}_{0}, k_{0}\right)$ is $B C F$.

Assumption 2b (Acyclicity Assumption) The influenced uncontrollable subnet of the initial singular G$\operatorname{MEC}\left(\boldsymbol{w}_{0}, k_{0}\right)$ is acyclic.

Assumption 2 includes two closely related sub-assumptions on the net structure, i.e., the influenced uncontrollable subnet is an acyclic BCF net. The class of BCF nets strictly includes marked graphs and assembly-flow systems [39]. In a BCF net, the tokens in a place $p$ can be uncontrollably injected only from its unique upstream uncontrollable transition. On the other hand, the assumption that the uncontrollable subnet is acyclic is a widely used assumption in the supervisory framework of Petri nets.

Example 3 In the net in Figure 7 with $T_{u}=\left\{t_{1}, t_{2}, t_{3}, t_{4}\right\}$, its uncontrollable subnet $N$ contains all places and transitions except $t_{c 1}, t_{c 2}, t_{c 3}$, and $t_{c 4}$ and it is backward-conflict-free.

BCF nets can model both conflicts (e.g., two or more workflows share the same source buffer) and synchronizations (e.g., two or more products are assembled) in the working flow systems. It is a widely studied 


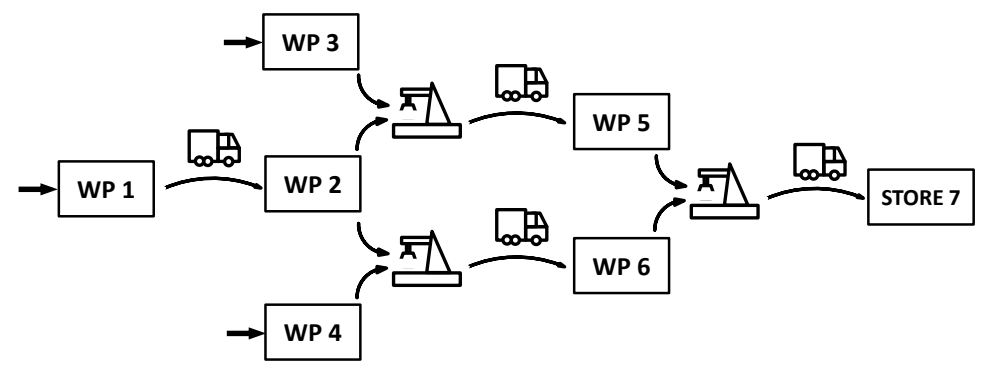

Figure 6: An automated assembly system.

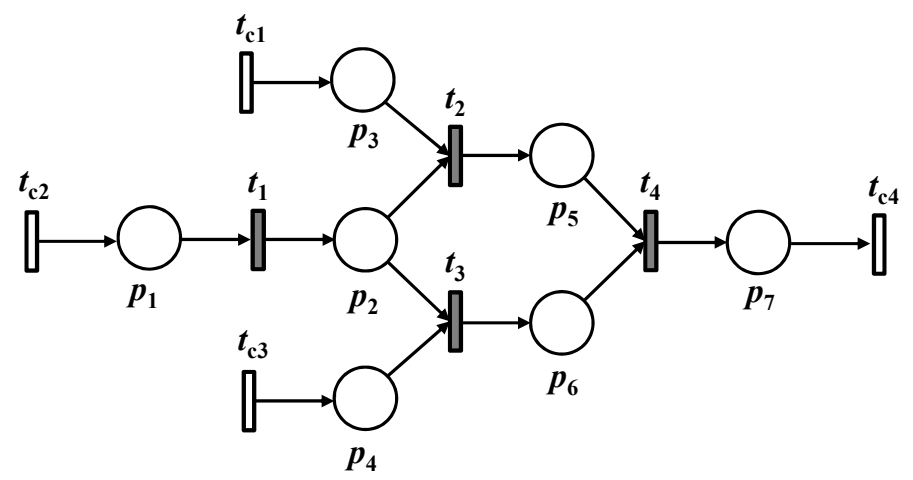

Figure 7: The Petri net model of the system in Figure 6.

subclass of Petri nets since many real systems (e.g., assembly workflows discussed in [39] and backwardconflict-free-choice-nets in [20]) can be modeled by it. In particular, for uncontrollable subnets that are backward-conflict-free-choice, by the technique in [20] an online feedback control logic with polynomial complexity can be obtained. On the other hand, the acyclic condition is reasonable since an uncontrollable loop may unstoppably consume incoming resources or cause an overflow.

Both Assumptions 1 and 2 can be immediately verified by the inspection of the net structure and the constraint structure. The two aforementioned assumptions are sufficient to rule out the GMEC inflation, which will be shown in the next section. Moreover, the following example shows that the GMEC inflation may occur if either of the two assumptions is not satisfied.

Example 4 Consider the following two cases: (1) the net in Figure 4(a) and an initial GMEC $\left(\boldsymbol{w}_{0}^{\prime}, k_{0}^{\prime}\right) \equiv$ $M\left(p_{5}\right) \leq k_{0}$ which does not satisfy Assumption 2; (2) the net in Figure 4(b) and an initial GMEC $\left(\boldsymbol{w}_{0}^{\prime \prime}, k_{0}^{\prime \prime}\right) \equiv$ $M\left(p_{3}\right)+M\left(p_{4}\right) \leq k_{0}$ which does not satisfy Assumption 1. In both cases the admissible marking sets are identical to that in Example 1, which suffers from the GMEC inflation. 


\subsection{Operations of Singular GMECs}

In this subsection we define two operations on singular GMECs, which will be used to build an algorithm in Section V. We point out that these operations can also be applied to singular GMECs in non-BCF nets. However, BCF nets have some special properties that will be used to guarantee the singularity of GMECs during the transformation procedure.

Definition 8 Given a singular GMEC $(\boldsymbol{w}, k)$ with root place $p_{x}$ and injection transition $t_{x}$ such that ${ }^{\bullet} t_{x}=$ $\left\{p_{1}, \ldots, p_{r}\right\}$, the expansion set of $(\boldsymbol{w}, k)$ is defined as $E_{(\boldsymbol{w}, k)}=\left\{\left(\boldsymbol{w}_{i}, k_{i}\right), 1 \leq i \leq r\right\}$, in which each $\left(\boldsymbol{w}_{i}, k_{i}\right)$ is computed by the following operations:

$$
\left\{\begin{array}{l}
w_{i}(p)=w\left(p_{x}\right)=1, \text { if } p=p_{i} \\
w_{i}(p)=w(p), \text { otherwise } \\
k_{i}=k
\end{array}\right.
$$

In brief, for each place $p_{i}$ in $t_{x}$ a new $\operatorname{GMEC}\left(\mathbf{w}_{i}, k_{i}\right)$ is put in $E_{(\mathbf{w}, k)}$ in which the weight of $p_{i}$ is increased to 1, i.e., the weight of $p_{x}$. The physical interpretation of the expansion operation is to backpropagate the tokens of its support to previous places through uncontrollable transitions. The following proposition shows that in an acyclic BCF net, given a singular $(\mathbf{w}, k)$, all GMECs in $E_{(\mathbf{w}, k)}$ are singular.

Proposition 5 Given a singular GMEC $(\boldsymbol{w}, k)$ with root place $p_{x}$ and injection transition $t_{x}$ with $\left|{ }^{\bullet} t_{x}\right|=r$, if its influenced uncontrollable subnet is acyclic and BCF, then all GMECs $\left(\boldsymbol{w}_{i}, k_{i}\right)$ in $E_{(w, k)}$ are singular.

Proof: Consider the arbitrary $i$-th GMEC $\left(\mathbf{w}_{i}, k_{i}\right)$ in $E_{(\mathbf{w}, k)}$.

(1) Since $|\mathbf{w}| \subset\left(p_{x}^{\bullet}\right)^{\infty}$ and $p_{x} \in\left(p_{i}^{\bullet}\right)^{\infty}$, we have $\left|\mathbf{w}_{i}\right|=|\mathbf{w}| \cup\left\{p_{i}\right\} \subset\left(p_{i}^{\bullet}\right)^{\infty}$ and by Definition 4 its unique root place is $p_{i}$.

(2) Since $(\mathbf{w}, k)$ is singular and the only weight change from $\mathbf{w}$ to $\mathbf{w}_{i}$ is that of $p_{i}: w_{i}\left(p_{i}\right)=w\left(p_{x}\right)=1$, the firing of any $t \notin \bullet p_{i}$ will not increase the token count of $\left(\mathbf{w}_{i}, k_{i}\right)$. By considering that the net is BCF, there exists at most one transition $t_{z} \in p_{i}$ such that $\mathbf{w}_{i}^{T} \cdot C\left(\cdot, t_{z}\right)>0$.

(3) Since the net is acyclic and $p_{i}$ is the root place of $\left(\mathbf{w}_{i}, k_{i}\right),{ }^{\bullet} t_{z} \cap\left|\mathbf{w}_{i}\right|=\emptyset$ holds. If $\mathbf{w}_{i}^{T} \cdot C\left(\cdot, t_{z}\right)>0$, then $t_{z}^{\bullet} \cap\left|\mathbf{w}_{i}\right|=\left\{p_{i}\right\}$ since $p_{i}$ is the root place of $\left(\mathbf{w}_{i}, k_{i}\right)$ and the net is BCF. Hence Condition 3 in Definition 5 is satisfied. By the three arguments, $\left(\mathbf{w}_{i}, k_{i}\right)$ is singular. 
Then we define the composition of two singular GMECs. Two GMECs are composable if they are both singular and share the same root place.

Definition 9 Given two singular GMECs $\left(\boldsymbol{w}_{a}, k_{a}\right)$ and $\left(\boldsymbol{w}_{b}, k_{b}\right)$ which share the same root place $p_{x}$, their composition GMEC $\left(\boldsymbol{w}_{a b}, k_{a b}\right)$ is computed by the following operations:

$$
\left\{\begin{array}{l}
w_{a b}(p)=1, \text { if } p=p_{x} \\
w_{a b}(p)=w_{a}(p)+w_{b}(p), \text { otherwise } \\
k_{a b}=\left[\left(k_{a}+1\right)+\left(k_{b}+1\right)\right]-1
\end{array}\right.
$$

Note that two singular GMECs are composable only if they have the same root place, and they would have the same injection transition if it exists. The graphic illustration of GMEC expansion and composition is shown in Figure 8.

Theorem 1 Given an acyclic net $N$ and a set of nonnegative singular GMECs, any GMEC that can be obtained by the expansion and composition operations has a unique root place.

Proof: The uniqueness of the root place for any GMEC obtained by the expansion follows from the proof of Proposition 5. For the composition, $\left|\mathbf{w}_{a b}\right| \subset\left(p_{x}^{\bullet}\right)^{\infty}$ holds since $\left|\mathbf{w}_{a b}\right|=\left|\mathbf{w}_{a}\right| \cup\left|\mathbf{w}_{b}\right|$ while both $\left|\mathbf{w}_{a}\right|$ and $\left|\mathbf{w}_{b}\right|$ are subsets of $\left(p_{x}^{\bullet}\right)^{\infty}$.

The physical interpretation of the composition operation is not straightforward. It characterizes the token conflict situation in place $p_{x}$ if $p_{x}$ is a conflict place, which help us to establish the algorithm in the next section. Specifically, if a marking $M$ satisfies the composed GMEC $\left(\mathbf{w}_{a b}, k_{a b}\right)$, then any marking uncontrollably reachable from $M$ cannot violate $\left(\mathbf{w}_{a}, k_{a}\right)$ and $\left(\mathbf{w}_{b}, k_{b}\right)$ simultaneously. Moreover, for arbitrary two singular composable GMECs, their composed GMEC is not always singular. However, in the next section we show that by proper ordering the expanding and composing sequence the singularity can be kept throughout a transformation algorithm.

\subsection{Problem Statement}

Now we state the problem to be studied in the remaining part of this paper.

Problem 2 Given a Petri net $N_{0}$ and a GMEC $\left(\boldsymbol{w}_{0}, k_{0}\right) \equiv M\left(p_{0}\right) \leq k_{0}$ that satisfies Assumptions 1 and 2, determine an equivalent controllable OR-GMEC $W$ such that $\mathcal{L}_{W}=\mathcal{A}_{\left(w_{0}, k_{0}\right)}$. 

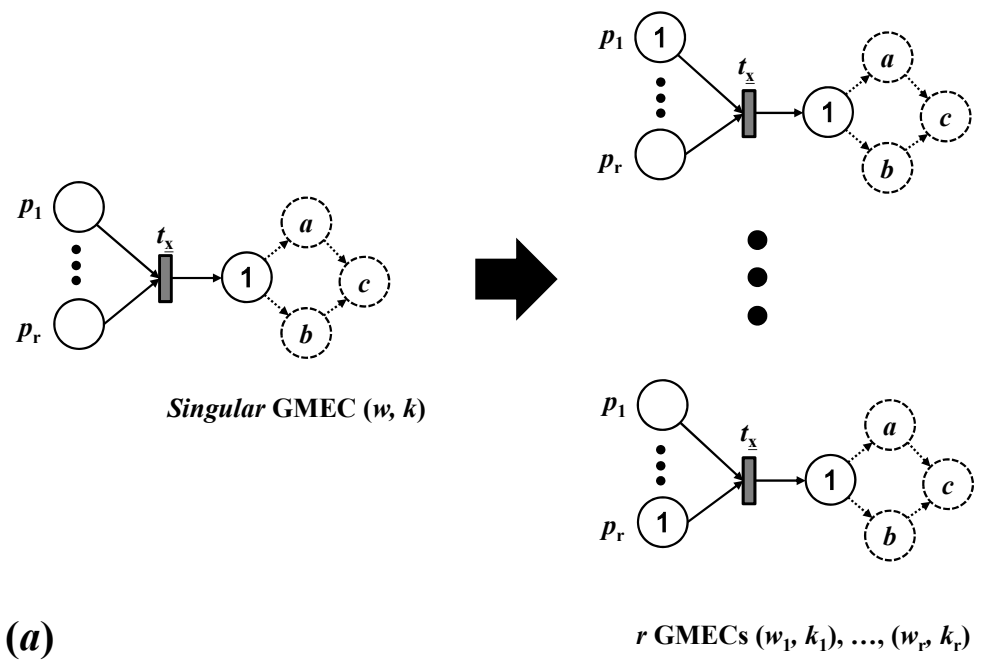

(a)

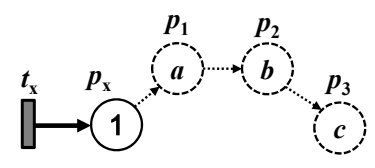

Singular GMEC $\left(w_{b}, k_{b}\right)$
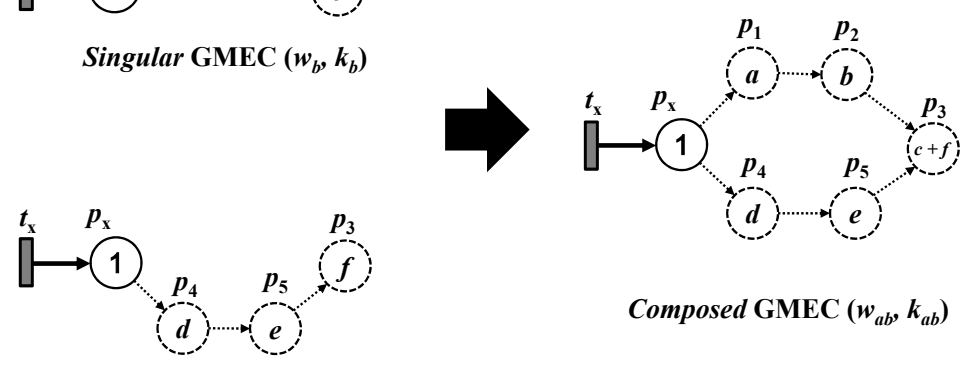

(b) Singular GMEC $\left(w_{b}, k_{b}\right)$

Figure 8: The graphic illustration of singular GMEC expansion (a) and composition (b). The number in each place represents its weight, i.e., $w(p)$. 
Example 5 Consider again the net in Figure 7 with the initial GMEC $\left(\boldsymbol{w}_{0}, k_{0}\right)=([0,0,0,0,0,0,1], 1)$, i.e., $M\left(p_{7}\right) \leq 1$. This GMEC is singular and its influenced uncontrollable subnet contains all places and transitions except $t_{c 1}, t_{c 2}, t_{c 3}$, and $t_{c 4}$ and it is acyclic and backward-conflict-free.

\section{GMEC Transformation in Acyclic BCF Uncontrollable Subnets}

\subsection{Algorithm}

In this section, we present Algorithm 1 which computes an OR-GMEC $W$ from a given singular GMEC $\left(\mathbf{w}_{0}, k_{0}\right)$. We claim that $W$ is the solution of Problem 2, i.e., $\mathcal{L}_{W}=\mathcal{A}_{\left(\mathbf{w}_{0}, k_{0}\right)}$.

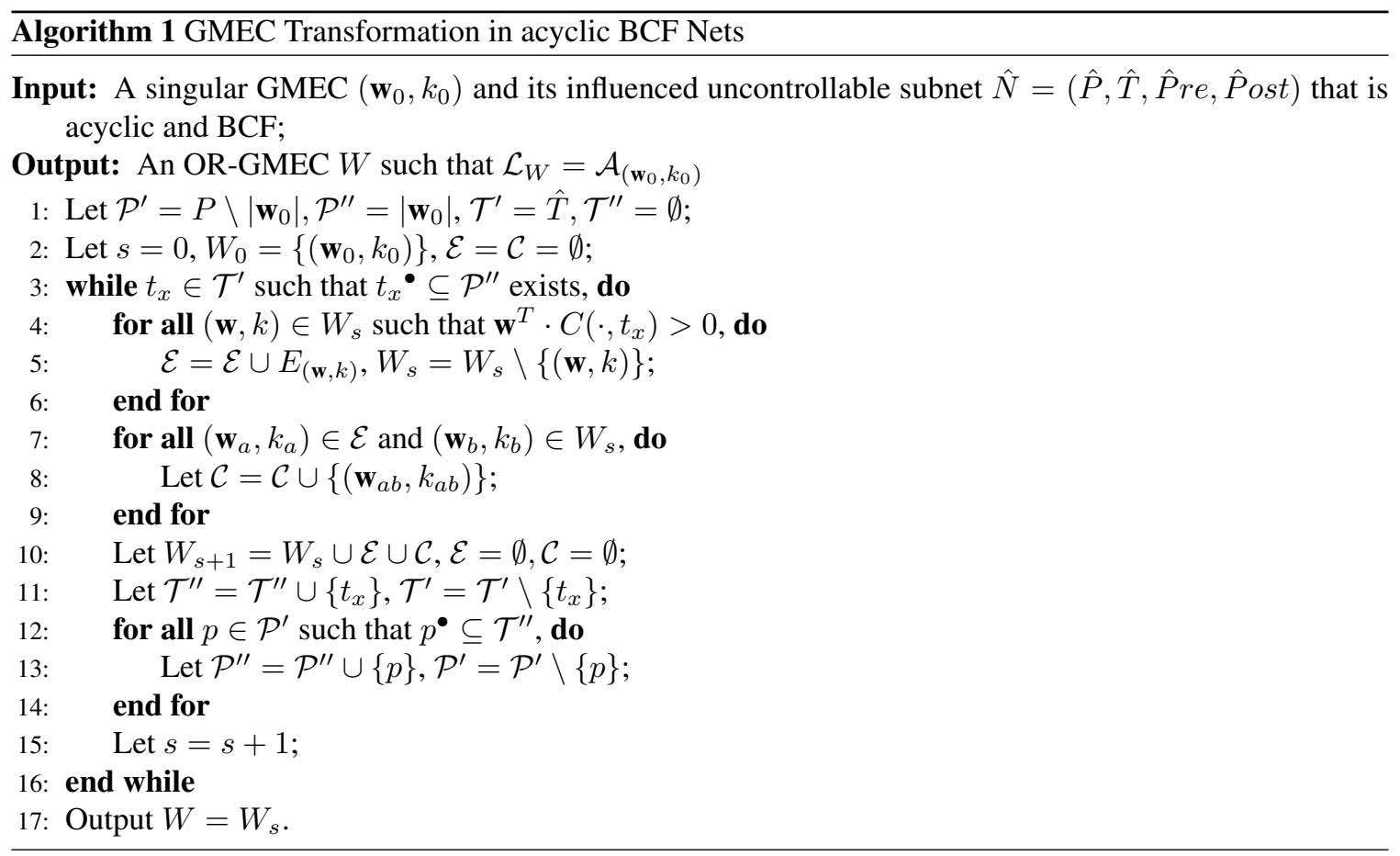

Algorithm 1 works as follows. In the initialization stage (Steps 1 and 2) four sets $\mathcal{P}^{\prime}, \mathcal{P}^{\prime \prime}, \mathcal{T}^{\prime}$, and $\mathcal{T}^{\prime \prime}$ are defined to record the current state of places and transitions, where $\mathcal{P}^{\prime} / \mathcal{T}^{\prime}$ and $\mathcal{P}^{\prime \prime} / \mathcal{T}^{\prime \prime}$ denote the unchecked and checked sets of places/transitions, respectively. Initially all transitions are put into the unchecked transition set $\mathcal{T}^{\prime}$, and the support places of $\mathbf{w}$ are put into the checked place set $\mathcal{P}^{\prime \prime}$ while other places are put into the unchecked place set $\mathcal{P}^{\prime}$. Step 2 initializes two temporary sets for GMECs $\mathcal{E}$ and $\mathcal{C}$.

Step 3 to 16 compose the iteration cycle. The iteration process is like a step-by-step analysis of $\hat{N}$ backward. At each iteration, an unchecked transition $t_{x}$ is picked from $\mathcal{T}^{\prime}$ in Step 3. The selection of transition $t_{x}$ is not random but follows the rule: a transition $t$ can be picked only if all its output places (i.e., all places in $t^{\bullet}$ ) are checked. We note that this rule is to ensure the singularity of all GMECs in $W_{s}$. Then 


\begin{tabular}{c|c|c|c|c|c|c}
\hline$s$ & Pick & $\mathcal{P}^{\prime}$ & $\mathcal{P}^{\prime \prime}$ & $\mathcal{T}^{\prime}$ & $\mathcal{T}^{\prime \prime}$ & $W_{s}$ \\
\hline 0 & - & $p_{1}, p_{2}, p_{3}, p_{4}, p_{5}, p_{6}$ & $p_{7}$ & $t_{1}, t_{2}, t_{3}, t_{4}$ & $\emptyset$ & $c_{0}$ \\
\hline 1 & $t_{4}$ & $p_{1}, p_{2}, p_{3}, p_{4}$ & $p_{5}, p_{6}, p_{6}$ & $t_{1}, t_{2}, t_{3}$ & $t_{4}$ & $c_{1}, c_{2}$ \\
\hline 2 & $t_{2}$ & $p_{1}, p_{2}, p_{4}$ & $p_{3}, p_{5}, p_{6}, p_{7}$ & $t_{1}, t_{3}$ & $t_{2}, t_{4}$ & $c_{2}, c_{3}, c_{4}$ \\
\hline 3 & $t_{3}$ & $p_{1}$ & $p_{2}, p_{3}, p_{4}, p_{5}, p_{6}, p_{7}$ & $t_{1}$ & $t_{2}, t_{3}, t_{4}$ & $c_{3}, c_{4}, c_{5}, c_{6}, c_{7}$ \\
\hline 4 & $t_{1}$ & $\emptyset$ & $p_{1}, p_{2}, p_{3}, p_{4}, p_{5}, p_{6}, p_{7}$ & $\emptyset$ & $t_{1}, t_{2}, t_{3}, t_{4}$ & $c_{3}, c_{6}, c_{8}, c_{9}, c_{10}$ \\
\hline
\end{tabular}

Table 1: Iteration steps of Example 6.

by Step 4 for each GMEC $(\mathbf{w}, k) \in W_{s}$ such that $\mathbf{w}^{T} \cdot C\left(\cdot, t_{x}\right)>0$ (i.e., the firing of $t_{x}$ will increase the token count of $(\mathbf{w}, k))$, its expansion set $E_{(\mathbf{w}, k)}$ is computed and put in $\mathcal{E}$ to be further treated, while $(\mathbf{w}, k)$ is removed from $W_{s}$.

Step 7 checks GMECs in $\mathcal{E}$ and $W_{s}$. If there exist $\left(\mathbf{w}_{a}, k_{a}\right) \in \mathcal{E}$ and $\left(\mathbf{w}_{b}, k_{b}\right) \in W_{s}$ which are composable, then their composed GMEC $\left(\mathbf{w}_{a b}, k_{a b}\right)$ is generated ${ }^{5}$ and put in $\mathcal{C}$. Step 10 computes $W_{s+1}$ as the union of $W_{s}, \mathcal{E}$ and $\mathcal{C}$. At the end of this iteration, $t_{x}$ is moved to $\mathcal{T}^{\prime \prime}$ as it has been checked, and in Step 12 a place $p \in \mathcal{P}^{\prime}$ is moved to $\mathcal{P}^{\prime \prime}$ if all transitions in $p^{\bullet}$ are checked. Note that Step 12 and Step 3 imply that a transition $t$ cannot be picked until all transitions in the downstream of $t$ (i.e., $\left(t^{\bullet}\right)^{\infty}$ ) are checked, since the influenced uncontrollable subnet is acyclic. Another iteration starts until there is no transition $t_{x}$ which could increase the token counts of GMECs in $W_{s}$.

\subsection{An Illustrative Example}

In this subsection we present an example to illustrate Algorithm 1. To simplify the notation we use $c_{(\cdot)}$ to denote $\left(\mathbf{w}_{(\cdot)}, k_{(\cdot)}\right)$.

Example 6 Let us consider the Petri net in Figure 7 that models the automated assembly system in Figure 6. Suppose that the storehouse has a maximum capacity of $k_{0}$, and hence the initial GMEC to be enforced is $c_{0}=\left(\boldsymbol{w}_{0}, k_{0}\right)=\left([0,0,0,0,0,0,1], k_{0}\right)$, i.e., $M\left(p_{7}\right) \leq k_{0}$. The entire iteration process is listed in Table 1. In $W_{0}$ there is only one constraint $c_{0}$ :

$$
c_{0}: M\left(p_{7}\right) \leq k_{0}
$$

In the first iteration, only transition $t_{4}$ could be picked. In Step 5, $c_{0}$ is substituted by $E_{c_{0}}$ consisting of two new constraints:

\footnotetext{
${ }^{5}$ If $\left(\mathbf{w}_{a}, k_{a}\right)$ and $\left(\mathbf{w}_{b}, k_{b}\right)$ are not composable, then no composed GMEC is generated, i.e., $\mathcal{C}$ remains unchanged.
} 


$$
\left\{\begin{array}{l}
c_{1}: M\left(p_{5}\right)+M\left(p_{7}\right) \leq k_{0} \\
c_{2}: M\left(p_{6}\right)+M\left(p_{7}\right) \leq k_{0}
\end{array}\right.
$$

Then $W_{1}=\left\{c_{1}, c_{2}\right\}$ is obtained by Step 10. In Step $11 t_{4}$ is moved to $\mathcal{T}^{\prime \prime}$. In Step $12 p_{5}$ and $p_{6}$ are moved to $\mathcal{P}^{\prime \prime}$. In the second iteration either $t_{2}$ or $t_{3}$ can be picked. Suppose that $t_{2}$ is picked. Since $t_{2}$ is the injection transition of $c_{1}, c_{1}$ is substituted by:

$$
\left\{\begin{array}{l}
c_{3}: M\left(p_{3}\right)+M\left(p_{5}\right)+M\left(p_{7}\right) \leq k_{0} \\
c_{4}: M\left(p_{2}\right)+M\left(p_{5}\right)+M\left(p_{7}\right) \leq k_{0}
\end{array}\right.
$$

Then we have $W_{2}=\left\{c_{2}, c_{3}, c_{4}\right\}$ and $t_{2}$ is moved to $\mathcal{T}^{\prime \prime}$. Since $p_{2}^{\bullet} \nsubseteq \mathcal{T}^{\prime \prime}$, $p_{2}$ remains in $\mathcal{P}^{\prime}$ (since a GMEC composition will be taken in some further iteration(s)). Therefore only $p_{3}$ is moved to $\mathcal{P}^{\prime \prime}$.

In the third iteration, since $p_{2} \notin \mathcal{P}^{\prime \prime}, t_{1}$ cannot be picked. Hence $t_{3}$ is picked and $c_{2}$ is substituted by $c_{5}$ and $c_{6}$ :

$$
\left\{\begin{array}{l}
c_{5}: M\left(p_{2}\right)+M\left(p_{6}\right)+M\left(p_{7}\right) \leq k_{0} \\
c_{6}: M\left(p_{4}\right)+M\left(p_{6}\right)+M\left(p_{7}\right) \leq k_{0}
\end{array}\right.
$$

At this moment, $c_{5}$ and $c_{4}$ are composable at $p_{2}$. Therefore Step 8 is triggered and a new composition GMEC $c_{7}$ is added:

$$
c_{7}: M\left(p_{2}\right)+M\left(p_{5}\right)+M\left(p_{6}\right)+2 M\left(p_{7}\right) \leq 2 k_{0}+1
$$

Now $W_{3}=\left\{c_{3}, c_{4}, c_{5}, c_{6}, c_{7}\right\}$ is obtained and $p_{2}$ is moved to $\mathcal{P}^{\prime \prime}$. In the final iteration, $t_{1}$ is picked and $c_{4}, c_{5}$ and $c_{7}$ are substituted by $c_{8}, c_{9}$ and $c_{10}$, respectively:

$$
\left\{\begin{aligned}
c_{8}: M\left(p_{1}\right)+M\left(p_{2}\right)+M\left(p_{5}\right)+M\left(p_{7}\right) & \leq k_{0} \\
c_{9}: M\left(p_{1}\right)+M\left(p_{2}\right)+M\left(p_{6}\right)+M\left(p_{7}\right) & \leq k_{0} \\
c_{10}: M\left(p_{1}\right)+M\left(p_{2}\right)+M\left(p_{5}\right)+M\left(p_{6}\right)+ & 2 M\left(p_{7}\right) \\
& \leq 2 k_{0}+1
\end{aligned}\right.
$$

The algorithm ends and outputs $W_{4}=\left\{c_{3}, c_{6}, c_{8}, c_{9}, c_{10}\right\}$ that is an OR-GMEC containing five single 


\section{Correctness of the GMEC Transformation Method}

Before formally showing the correctness of the algorithm, we provide a short roadmap of the proof which is built on two observations. First, by the assumption that the initial GMEC is singular and the uncontrollable net is acyclic and BCF, it holds that at arbitrary iteration step $s$ of Algorithm 1, all GMECs in $W_{s}$ are singular. Hence when Algorithm 1 ends, all GMECs in $W$ are singular with no injection transitions, which implies that all GMECs in the output $W$ are controllable. Second, at any iteration step $s$ of Algorithm 1, by the singularity of GMECs in $W_{s-1}$ the admissible marking sets $\mathcal{A}_{W_{s}}$ and $\mathcal{A}_{W_{s+1}}$ are identical. In the sequel when we say "at the beginning of the $s$-th iteration step" and "at the end of $s$-th iteration step", we refer to Step 3 and Step 14 of the $s$-th iteration, respectively.

\subsection{Singularity of GMECs in $W_{s}$}

The following proposition reveals the relationship between the GMEC $\left(\mathbf{w}_{a b}, k_{a b}\right)$ composed by $\left(\mathbf{w}_{a}, k_{a}\right) \in$ $E_{\left(\mathbf{w}_{a_{0}}, k_{a_{0}}\right)}$ and $\left(\mathbf{w}_{b}, k_{b}\right) \in W_{s}$ and its ancestor $\left(\mathbf{w}_{a_{0}}, k_{a_{0}}\right)$.

Proposition 6 At each iteration step of Algorithm 1, if $\left(\boldsymbol{w}_{a}, k_{a}\right) \in E_{\left(\boldsymbol{w}_{a_{0}}, k_{a_{0}}\right)}$ is composed with $\left(\boldsymbol{w}_{b}, k_{b}\right)$, then $\boldsymbol{w}_{a b}=\boldsymbol{w}_{a_{0}}+\boldsymbol{w}_{b}$

Proof: Let $p_{x}$ be the root place of $\left(\mathbf{w}_{a}, k_{a}\right)$. Then $w_{a b}(p)=w_{a_{0}}(p)+w_{b}(p)$ holds by the fact that $w_{a_{0}}\left(p_{x}\right)=0$ and $w_{a_{0}}(p)=w_{a}(p)$ for all $p \neq p_{x}$.

Proposition 7 At each iteration step of Algorithm $1, t \in \mathcal{T}^{\prime \prime}$ implies that $\forall x \in\left(t^{\bullet}\right)^{\infty}, x \in \mathcal{T}^{\prime \prime} \cup \mathcal{P}^{\prime \prime}$.

Proof: Trivial, since a place $p$ is put into $\mathcal{P}^{\prime \prime}$ only if $p^{\bullet} \subseteq \mathcal{T}^{\prime \prime}$, and a transition $t$ is put into $\mathcal{T}^{\prime \prime}$ only if $t^{\bullet} \subseteq \mathcal{P}^{\prime \prime}$

Proposition 8 At the end of each s-th iteration of Algorithm 1, for an arbitrary GMEC $(\boldsymbol{w}, k)$ in $W_{s}$, $(\bullet t \cap$ $\left.|\boldsymbol{w}| \neq \emptyset, t^{\bullet} \cap|\boldsymbol{w}| \neq \emptyset\right) \Rightarrow\left(t \in \mathcal{T}^{\prime \prime}\right)$.

Proof: We prove this by contradiction. Suppose that at the beginning of $s$-th iteration a transition $t_{x}$ is picked, and at the end of this iteration there exists a $\operatorname{GMEC}(\mathbf{w}, k)$ and a transition $t_{y} \in \mathcal{T}^{\prime}$ such that $\bullet t_{y} \cap|\mathbf{w}| \neq \emptyset$ and $t_{y}^{\bullet} \cap|\mathbf{w}| \neq \emptyset$. Since the net is BCF, it is trivial that $(\mathbf{w}, k)$ cannot be generated by 


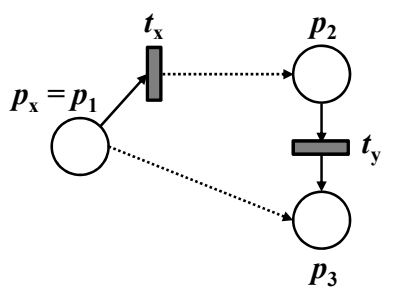

(a)

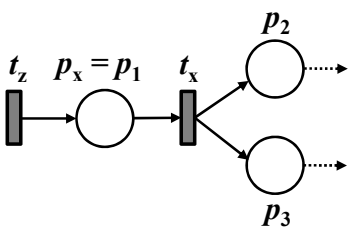

(b)

Figure 9: The example to illustrate the proofs of Proposition 8 and Theorem 2.

the expansion. Hence $(\mathbf{w}, k)$ must be composed by two singular GMECs $\left(\mathbf{w}_{a}, k_{a}\right)$ and $\left(\mathbf{w}_{b}, k_{b}\right)$ such that ${ }^{\bullet} t_{y} \cap\left|\mathbf{w}_{a}\right| \neq \emptyset, t_{y}^{\bullet} \cap\left|\mathbf{w}_{a}\right|=\emptyset,{ }^{\bullet} t_{y} \cap\left|\mathbf{w}_{b}\right|=\emptyset$, and $t_{y}^{\bullet} \cap\left|\mathbf{w}_{b}\right| \neq \emptyset$, which is illustrated in Figure 9(a) where $p_{1}, p_{2} \in\left|\mathbf{w}_{a}\right|$ and $p_{1}, p_{3} \in\left|\mathbf{w}_{b}\right|$. Hence $t_{y}$ is in the downstream of $t_{x}$, which is impossible since $t_{x}$ can only be picked after $t_{y}$ is checked. Therefore the statement holds.

Now we prove that all GMECs in $W_{s}$ are singular.

Theorem 2 At any iteration step s of Algorithm 1, all GMECs in $W_{s}$ are singular.

Proof: We prove this by induction.

(Base step) For $W_{0}=\left\{\left(\mathbf{w}_{0}, k_{0}\right)\right\},\left(\mathbf{w}_{0}, k_{0}\right)$ is singular by Assumption 1 .

(Induction Step) Assume that at the beginning of $s$-th iteration, all GMECs in $W_{s}$ are singular. Suppose that at this iteration a transition $t_{x} \in \mathcal{T}^{\prime}$ is picked. Since all GMECs in $W_{s}$ are singular, by Proposition 5 all new GMECs generated by the expansion operation are singular. Now we prove that all new GMECs generated by composition are also singular.

At this iteration, a composed $\left(\mathbf{w}_{a b}, k_{a b}\right)$ is generated by the following procedure: by picking $t_{x}$ some $\operatorname{GMEC}\left(\mathbf{w}_{a_{0}}, k_{a_{0}}\right)$ is substituted by $E_{\left(\mathbf{w}_{a_{0}}, k_{a_{0}}\right)}$ through $t_{x}$, and then some $\left(\mathbf{w}_{a}, k_{a}\right)$ in $E_{\left(\mathbf{w}_{a_{0}}, k_{a_{0}}\right)}$ is composed with $\left(\mathbf{w}_{b}, k_{b}\right)$ in $W_{s}$, producing the new constraint $\left(\mathbf{w}_{a b}, k_{a b}\right)$.

Since $\left(\mathbf{w}_{a}, k_{a}\right)$ and $\left(\mathbf{w}_{b}, k_{b}\right)$ are composable, the common injection transition $t_{z}$, if it exists, is unique. Since all input places of $t_{z}$ have a weight zero in $\mathbf{w}_{a}$ and $\mathbf{w}_{b}$, we have $\mathbf{w}_{a b}^{T} \cdot C\left(\cdot, t_{z}\right)=1$. Now let us consider an arbitrary transition $t \neq t_{z}$. Since both $\left(\mathbf{w}_{a}, k_{a}\right)$ and $\left(\mathbf{w}_{b}, k_{b}\right)$ are singular, $\mathbf{w}_{a}^{T} \cdot C(\cdot, t) \leq 0$ and $\mathbf{w}_{b}^{T} \cdot C(\cdot, t) \leq 0$ hold. Therefore $\left(\mathbf{w}_{a}+\mathbf{w}_{b}\right)^{T} \cdot C(\cdot, t) \leq 0$ that, by Proposition 6 , can be rewritten as:

$$
\begin{aligned}
\left(\mathbf{w}_{a}+\mathbf{w}_{b}\right)^{T} \cdot C(\cdot, t) & =\left(\mathbf{w}_{a_{0}}+\mathbf{w}_{b}\right)^{T} \cdot C(\cdot, t)+C\left(p_{x}, t\right) \\
& =\mathbf{w}_{a b}^{T} \cdot C(\cdot, t)+C\left(p_{x}, t\right) \leq 0
\end{aligned}
$$


Since the influenced uncontrollable subnet is assumed to be BCF and hence ordinary (see Definition 7), the only possibility to make $\mathbf{w}_{a b}^{T} \cdot C(\cdot, t)>0$ is that in Eq. (5) $C\left(p_{x}, t\right)=-1$ and $\mathbf{w}_{a_{0}} \cdot C(\cdot, t)=1$ hold. Since $\mathbf{w}_{a_{0}} \cdot C(\cdot, t)=1$, transition $t$ is the injection transition $t_{x}$ of $\left(\mathbf{w}_{a_{0}}, k_{a_{0}}\right)$, i.e., $t=t_{x}$, which is picked at this iteration. Since $\mathbf{w}_{b}^{T} \cdot C\left(\cdot, t_{x}\right)=0, p_{x} \in\left|\mathbf{w}_{b}\right|$ and $C\left(p_{x}, t\right)=-1$, we have $\bullet \bullet\left|\mathbf{w}_{b}\right| \neq \emptyset$ and $t^{\bullet} \cap\left|\mathbf{w}_{b}\right| \neq \emptyset$. However, by noting that $\left(\mathbf{w}_{b}, k_{b}\right)$ is inherited from $W_{s-1}$, by Proposition $8, t_{x}$ is already in $\mathcal{T}^{\prime \prime}$ at the beginning of this iteration and thus it cannot be picked. This contradiction excludes the possibility of $\mathbf{w}_{a b}^{T} \cdot C(\cdot, t)>0$.

As a result, (1) $\left(\mathbf{w}_{a b}, k_{a b}\right)$ has a unique root place $p_{x}$, (2) there exists at most one transition $t_{z}$ such that $\mathbf{w}_{a b}^{T} \cdot C\left(\cdot, t_{z}\right)>0$, and (3) $\mathbf{w}_{a b}^{T} \cdot C\left(\cdot, t_{z}\right)=1$ and $\bullet t_{z} \cap\left|\mathbf{w}_{a b}\right|=t_{z}^{\bullet} \cap\left|\mathbf{w}_{a b}\right|=\emptyset$. Hence $\left(\mathbf{w}_{a b}, k_{a b}\right)$ is singular by Definition 5 and the statement holds.

For a better understanding we briefly explain the induction step of Theorem 2. By Proposition 5 a GMEC obtained by the expansion of a singular GMEC is necessarily singular. Hence we need to exclude the possibility that a non-singular GMEC is composed by two singular GMECs. The only possibility to make $\mathbf{w}_{a b}^{T} \cdot C(\cdot, t)>0$ in Eq. (5) is illustrated in Figure 9(b): suppose that there exists a singular GMEC $\left(\mathbf{w}_{a_{0}}, k_{a_{0}}\right) \equiv M\left(p_{2}\right)+\cdots \leq k_{a_{0}}$. By selecting $t_{x}$, a new $\operatorname{GMEC}\left(\mathbf{w}_{a}, k_{a}\right) \equiv M\left(p_{1}\right)+M\left(p_{2}\right)+\cdots \leq k_{a}$ is obtained, which is composed with $\left(\mathbf{w}_{b}, k_{b}\right) \equiv M\left(p_{1}\right)+M\left(p_{3}\right)+\cdots \leq k_{b}$. However, since both $p_{1}$ and $p_{3}$ are already in $\left|\mathbf{w}_{b}\right|, t_{x}$ must be already checked and cannot be picked at this iteration. Therefore $\mathbf{w}_{a b}^{T} \cdot C(\cdot, t) \leq 0$ holds and the statement in Theorem 2 is true. From Theorem 2 and Proposition 4 we immediately have the following results.

Proposition 9 At any iteration step s of Algorithm 1, for arbitrary $(\boldsymbol{w}, k) \in W_{s}$ and $t \in \mathcal{T}^{\prime \prime}$, the firing of $t$ does not increase the token count of $(\boldsymbol{w}, k)$.

Proof: Since all GMECs in $W_{s}$ are singular, only the firing of its injection transition $t_{x} \in \boldsymbol{\bullet}^{\bullet} p_{x}$ can increase its token count. By the rule of selection, $t \in \mathcal{T}^{\prime \prime}$ implies $t \notin\left({ }^{\bullet} p_{x}\right)^{\infty}$, and hence the firing of $t$ does not increase its token count in forthcoming iterations.

Since Algorithm 1 ends after $|\hat{T}|$ iterations, i.e., all uncontrollable transitions in $\mathcal{T}^{\prime}$ are moved to $\mathcal{T}^{\prime \prime}$. Therefore we have the following result from Proposition 9.

Corollary 2 The OR-GMEC output by Algorithm 1 is controllable.

Moreover, the following proposition shows that the negative support of the initial GMEC remains unchanged during the transformation, and if two GMECs are composable by Algorithm 1, the firing of $t_{x}$ does not change the token count of their composed GMEC. 
Proposition 10 At any iteration step s of Algorithm $1,\left((\boldsymbol{w}, k) \in W_{s}, w(p)<0\right) \Leftrightarrow\left(w_{0}(p)<0\right)$.

Proof: This can be trivially proved by induction. At the first iteration, i.e, $s=1$, the statement holds. For the induction step, suppose that at the $\bar{s}$-th iteration the statement holds. Then at the $(\bar{s}+1)$-th iteration, any new $\operatorname{GMEC}\left(\mathbf{w}_{a}, k_{a}\right)$ obtained by the expansion of $\left(\mathbf{w}_{a_{0}}, k_{a_{0}}\right)$ has exactly the same negative support since the only weight change is the new root place in $\bullet t$ whose weight increases from 0 to 1 , and obviously a composed GMEC would have the identical negative support places if it is composed by two GMECs that have the same negative support places.

Proposition 11 At any iteration step s of Algorithm 1, if $\left(\boldsymbol{w}_{a}, k_{a}\right) \in E_{(\boldsymbol{w}, k)}$ and $\left(\boldsymbol{w}_{b}, k_{b}\right)$ are composable, then $\boldsymbol{w}_{a b}^{T} \cdot C\left(\cdot, t_{x}\right)=0$ and $\boldsymbol{w}_{b}^{T} \cdot C\left(\cdot, t_{x}\right)=-1$.

Proof: By Theorem 2 the firing of $t_{x}$ does not increase the token count of $\left(\mathbf{w}_{a b}, k_{a b}\right)$, since $\mathbf{w}_{a b}^{T}$. $C\left(\cdot, t_{x}\right)=\mathbf{w}_{a_{0}}^{T} \cdot C\left(\cdot, t_{x}\right)+\mathbf{w}_{b}^{T} \cdot C\left(\cdot, t_{x}\right)=1+\mathbf{w}_{b}^{T} \cdot C\left(\cdot, t_{x}\right) \leq 0$. If “ $<$ " holds, then $\mathbf{w}_{b}^{T} \cdot C\left(\cdot, t_{x}\right) \leq-2$. Notice that $t_{x}$ is the injection transition of $\left(\mathbf{w}_{a_{0}}, k_{a_{0}}\right)$. There does not exist a place $p \in t_{x}^{\bullet}$ such that $w_{a}(p) \geq 0$, and hence by Proposition $10 \forall p \in t_{x}^{\bullet}, w_{b}(p) \geq 0$ holds. Since the net is ordinary, $\bullet_{x} \cap\left|\mathbf{w}_{b}\right| \geq 2$ is true. This implies that some transition $t_{y}$ that satisfies ${ }^{\bullet} t_{y} \cap\left|\mathbf{w}_{b}\right| \neq \emptyset$ and $t_{y}^{\bullet} \cap\left|\mathbf{w}_{b}\right| \neq \emptyset$ is checked before $t_{x} \in\left(t_{y}^{\bullet}\right)^{\infty}$, which contradicts Proposition 7. Hence " $=$ " must hold, and $\mathbf{w}_{a b}^{T} \cdot C\left(\cdot, t_{x}\right)=0$ is true.

Since $\mathbf{w}_{a_{0}}^{T} \cdot C\left(\cdot, t_{x}\right)=1, \mathbf{w}_{a b}^{T} \cdot C\left(\cdot, t_{x}\right)=0$, and $\mathbf{w}_{a b}=\mathbf{w}_{a_{0}}+\mathbf{w}_{b}$, we have $\mathbf{w}_{b}^{T} \cdot C\left(\cdot, t_{x}\right)=-1$, which concludes the statement.

\subsection{The Invariance of Admissible Sets}

This subsection develops the key step to the final result. The following theorem claims that during the execution of Algorithm 1, the admissible marking set of each $W_{s}$ remains identical.

Theorem 3 In Algorithm 1, $\mathcal{A}_{W_{s+1}}=\mathcal{A}_{W_{s}}$.

Proof: To prove this theorem we prove that both $\mathcal{A}_{W_{s+1}} \subseteq \mathcal{A}_{W_{s}}$ and $\mathcal{A}_{W_{s+1}} \supseteq \mathcal{A}_{W_{s}}$ hold. At the beginning of each iteration $s$, a transition $t_{x} \in \mathcal{T}^{\prime}$ is picked and some GMECs $\left(\mathbf{w}_{a_{1}}, k_{a_{1}}\right), \ldots,\left(\mathbf{w}_{a_{r}}, k_{a_{r}}\right) \in$ $W_{s}$ are picked. In Step 5 their expansion sets are computed whose union is $\mathcal{E}$. If a $\operatorname{GMEC}\left(\mathbf{w}_{a}, k_{a}\right) \in \mathcal{E}$ and a $\operatorname{GMEC}\left(\mathbf{w}_{b}, k_{b}\right)$ in the remaining of $W_{s}$, denoted as $W_{s}^{\prime}$, is composable, a new GMEC $\left(\mathbf{w}_{a b}, k_{a b}\right)$ is generated and put into $\mathcal{C}$. Finally $W_{s+1}=W_{s}^{\prime} \cup \mathcal{E} \cup \mathcal{C}$.

$\left(\mathcal{A}_{W_{s+1}} \subseteq \mathcal{A}_{W_{s}}\right)$ We first show that $\mathcal{L}_{W_{s}} \supseteq \mathcal{L}_{W_{s+1}}$. Consider a marking $M$ which violates all GMEC in $W_{s}$. On the one hand, by the definition of the expansion operation, if $M$ violates $(\mathbf{w}, k)$ then $M$ violates all 
GMECs in $E_{(\mathbf{w}, k)}$. Hence $M$ violates all GMECs in $\mathcal{E}$. On the other hand, if $M$ violates both $\left(\mathbf{w}_{a}, k_{a}\right)$ and $\left(\mathbf{w}_{b}, k_{b}\right)$, then $M$ violates their composed GMEC $\left(\mathbf{w}_{a b}, k_{a b}\right)$. The reason is stated as follows. Suppose that $\left(\mathbf{w}_{a}, k_{a}\right)$ is in the expansion set of $\left(\mathbf{w}_{a_{0}}, k_{a_{0}}\right)$, i.e., $\left(\mathbf{w}_{a}, k_{a}\right) \in E_{\left(\mathbf{w}_{a_{0}}, k_{a_{0}}\right)}$. By the definition of the composition operation and Proposition 6, $\mathbf{w}_{a b}=\mathbf{w}_{a_{0}}+\mathbf{w}_{b}$ and $k_{a b}=k_{a_{0}}+k_{b}+1$ hold. Since $M$ violates $\left(\mathbf{w}_{a_{0}}, k_{a_{0}}\right)$ and $\left(\mathbf{w}_{b}, k_{b}\right), \mathbf{w}_{a_{0}}^{T} \cdot M \geq k_{a_{0}}+1$ and $\mathbf{w}_{b}^{T} \cdot M \geq k_{b}+1$ hold, which indicates that $\mathbf{w}_{a b}^{T} \cdot M \geq k_{a_{0}}+k_{b}+2>k_{a b}$, leading to the fact that $M$ violates all composed GMEC in $\mathcal{C}$. Hence $M \notin \mathcal{L}_{W_{s}}$ implies $M \notin \mathcal{L}_{W_{s+1}}$, and thus $\mathcal{L}_{W_{s}} \supseteq \mathcal{L}_{W_{s+1}}$.

For a marking $M \in \mathcal{A}_{W_{s+1}}, M$ would never evolve to a marking $M^{\prime} \notin \mathcal{L}_{W_{s+1}}$ by firing uncontrollable transitions. Since $\mathcal{L}_{W_{s}} \supseteq \mathcal{L}_{W_{s+1}}, M$ would never evolve to a marking $M^{\prime} \notin \mathcal{L}_{W_{s}}$ by firing uncontrollable transitions. This indicates $M \in \mathcal{A}_{W_{s}}$ and hence $\mathcal{A}_{W_{s+1}} \subseteq \mathcal{A}_{W_{s}}$.

$\left(\mathcal{A}_{W_{s+1}} \supseteq \mathcal{A}_{W_{s}}\right)$ We prove that if a marking $M_{0}$ is not in $\mathcal{A}_{W_{s+1}}$, i.e., $M_{0}$ would evolve to a marking $M$ violating all GMECs in $\mathcal{L}_{W_{s+1}}$ by firing only uncontrollable transitions, then from $M$ by repeatedly firing $t_{x}$ for enough times we reach a new marking $M^{\prime}$ which violates all GMECs in $W_{s}$.

Suppose that $M$ violates all GMECs in $\mathcal{L}_{W_{s+1}}$. Let $\left(\mathbf{w}_{a_{0}}, k_{a_{0}}\right)$ be the GMEC among those picked in Step 4 such that $\mathbf{w}_{a_{0}}^{T} \cdot M \leq k_{a_{0}}$ but with a minimum shortage. Let $\left(\mathbf{w}_{b}, k_{b}\right)$ be the GMEC in $W_{s}^{\prime}$ such that $\mathbf{w}_{b}^{T} \cdot M \geq k_{b}$ but with a minimum excess. We claim that by repeatedly firing $t_{x}$ for a certain number of times at $M$, we can always reach a new marking $M^{\prime}$ which simultaneously violates $\left(\mathbf{w}_{a_{0}}, k_{a_{0}}\right)$ and $\left(\mathbf{w}_{b}, k_{b}\right)$, i.e., $\mathbf{w}_{a_{0}}^{T} \cdot M^{\prime}>k_{a_{0}}, \mathbf{w}_{b}^{T} \cdot M^{\prime}>k_{b}$.

By contradiction, if this operation cannot be done, then at a certain moment a marking $M_{f}$ is reached while there exists at least one place $p_{i} \in t^{\bullet}$ that is empty. On the one hand, if $\mathbf{w}_{a_{0}}^{T} \cdot M_{f} \leq k_{a_{0}}, M^{\prime}$ does not violate $\left(\mathbf{w}_{a}, k_{a}\right)$ since the firing of $t_{x}$ does not change the token count of $\left(\mathbf{w}_{i}, k_{i}\right)$ corresponding to $p_{i}$ in the expansion set of $\left(\mathbf{w}_{a_{0}}, k_{a_{0}}\right)$. On the other hand, if $\mathbf{w}_{a_{0}}^{T} \cdot M_{f}=k_{a_{0}}+1$ (it is always possible since each firing of $t_{x}$ increases the token count of $\left(\mathbf{w}_{a_{0}}, k_{a_{0}}\right)$ by one due to Proposition 4$)$ and $\mathbf{w}_{b}^{T} \cdot M_{f} \leq k_{b}$, by

$$
\begin{aligned}
\mathbf{w}_{a b}^{T} \cdot M_{f} & =\left(\mathbf{w}_{a_{0}}+\mathbf{w}_{b}\right)^{T} \cdot M_{f} \\
& \leq k_{a_{0}}+1+k_{b} \\
& =k_{a b},
\end{aligned}
$$

$M_{f}$ satisfies $\left(\mathbf{w}_{a b}, k_{a b}\right)$. However, by Proposition 11, the firing of $t_{x}$ does not change the token count of $\left(\mathbf{w}_{a b}, k_{a b}\right)$, which also contradicts the fact that $M^{\prime}$ is not in $\mathcal{L}_{W_{s+1}}$. By the selection rule of $\left(\mathbf{w}_{a_{0}}, k_{a_{0}}\right)$ and $\left(\mathbf{w}_{b}, k_{b}\right)$, all GMECs in $W_{s}$ are also violated at $M^{\prime}$.

Since $M^{\prime}$ can always be reached and $M^{\prime} \notin \mathcal{L}_{W_{s}}$, we have $M_{0} \notin \mathcal{A}_{W_{s}}$. Hence $\mathcal{A}_{W_{s+1}} \supseteq \mathcal{A}_{W_{s}}$. 


\subsection{Final Result}

Finally, by the series of results above, we can state the main result of this paper.

Theorem 4 The OR-AND GMEC W output by Algorithm 1 satisfies $\mathcal{L}_{W}=\mathcal{A}_{\left(w_{0}, k_{0}\right)}$.

Proof: First, the initial GMEC $\left(\mathbf{w}_{0}, k_{0}\right)$ is singular. Since the net is acyclic and BCF, according to Theorem 2, all GMECs in $W_{s}$ are singular in all iterations. By repeatedly applying Theorem $3, \mathcal{A}_{W}=$ $\mathcal{A}_{\left(\mathrm{w}_{0}, k_{0}\right)}$ holds. According to Corollary $2, \mathcal{L}_{W}=\mathcal{A}_{W}$. Hence $\mathcal{L}_{W}=\mathcal{A}_{\left(\mathrm{w}_{0}, k_{0}\right)}$.

Let us provide some motivation for the GMEC inflation when Assumptions 1 and/or 2 are unsatisfied. If the net is not $\mathrm{BCF}$, then during the transformation the resulting GMEC would not be singular. For a nonsingular GMEC, Proposition 4 does not hold, i.e., the firing of $t$ may change its token count by $q>1$. This makes $\left(\mathcal{A}_{W_{s+1}} \supseteq \mathcal{A}_{W_{s}}\right)$ in Theorem 3 unfounded. In fact, from $M$ by firing $t_{x}$ it may not be possible to reach a marking $M_{f}$ such that $\mathbf{w}_{a_{0}}^{T} \cdot M_{f}=k_{a_{0}}+1$, since its token count can only vary by a multiple of $q$. This integer-discontinuity would fragment the inadmissible marking set and hence cause the GMEC inflation.

Now we discuss the complexity of Algorithm 1. At each iteration step of Algorithm 1, a transition in $\mathcal{T}^{\prime}$ is picked. Hence Algorithm 1 terminates after at most $|\hat{T}|$ iterations. However, in the worst case the number of single GMECs in the set $W$ may be exponential with respect to that of conflict-synchronization structures in the system. The number of GMECs in the final $W$ depends on the conflict-synchronization structure in the influenced uncontrollable subnet $\hat{N}$. Specifically, the alternation of conflict places and synchronization transitions in one path would greatly increase the number of GMECs in $W$, while a large uncontrollable subnet with few such structures would have a very simple $W$. However, since both the expansion and the composition operations simply involve a vector addition, Algorithm 1 is usually efficient, and in many practical cases the number of GMECs in $W$ remains in an acceptable scale.

Example 7 (Ex. 6 Continued) Let us again consider the Petri net in Figure 7 and the initial GMEC to be enforced $c_{0}: M\left(p_{7}\right) \leq k_{0}$. As discussed in Example 6, by Algorithm 1 we obtain an OR-GMEC $W=$ $\left\{c_{3}, c_{6}, c_{8}, c_{9}, c_{10}\right\}$. One can verify that the OR-AND GMEC is controllable and maximally permissive, i.e., $\mathcal{L}_{W}=\mathcal{A}_{\left(w_{0}, k_{0}\right)}$. We also note that the GMEC inflation does not occur since the output OR-GMECW always contain five single GMECs regardless the value of $k_{0}$.

At the end of this paper we point out that Sections V and VI focus on the case that the initial legal marking set to be enforced is defined by a single GMEC $\left(\mathbf{w}_{0}, k_{0}\right)$. The method can be generalized to the case in which the initial legal marking set is defined by an OR-AND GMEC $W_{0}$. In fact, for each single GMEC $\left(\mathbf{w}_{i j}, k_{i j}\right)$ in $W_{0}$, Algorithm 1 can be carried out to convert it into an OR-GMEC $W_{O R, i j}$ such that $\mathcal{L}_{W_{O R, i j}}=\mathcal{A}_{\left(\mathbf{w}_{i j}, k_{i j}\right)}$. Hence the admissible marking set $\mathcal{A}_{W_{0}}$ can be defined by the linear constraint $\bigvee_{i} \bigwedge_{j} W_{O R, i j}$ that can be 
converted to its equivalent disjunctive normal form. As a result, the admissible marking set $\mathcal{A}_{W_{0}}$ is defined by an OR-AND GMEC.

\section{Conclusion}

In this paper the problem of GMEC transformation in Petri nets is studied. The contribution of this work is two-fold. First, the properties of the admissible marking set of a given GMEC specification are studied. The admissible marking set may not be represented by an OR-AND GMEC in general, and it may be too complex to be practically enforced due to the occurrence of the GMEC inflation phenomenon. We characterize two structural assumptions for ruling out the GMEC inflation, i.e, the singularity of the initial GMEC and the backward-conflict-freeness of its influenced subnet. Second, if these conditions are satisfied, an effective GMEC transformation method is proposed to compute the admissible marking set. As far as we know, it is the first work to preform the GMEC transformation in Petri nets containing both conflicts and synchronizations.

\section{References}

[1] P. J. Ramadge and W. M. Wonham, "The control of discrete event systems," Proceedings of IEEE, vol. 77, no. 1, pp. 81-98, 1989.

[2] Y. Li and W. M. Wonham, "Control of vector discrete-event systems. I. the base model," IEEE Transactions on Automatic Control, vol. 38, no. 8, pp. 1214-1227, 1993.

[3] J. Ezpeleta, J. M. Colom, and J. Martinez, "A Petri net based deadlock prevention policy for flexible manufacturing systems," IEEE Transactions on Robotics and Automation, vol. 11, no. 2, pp. 173-184, 1995.

[4] Z. W. Li and M. C. Zhou, "Elementary siphons of Petri nets and their application to deadlock prevention in flexible manufacturing systems," IEEE Transactions on Systems, Man, and Cybernetics, Part A, vol. 34, no. 1, pp. 38-51, 2004.

[5] Z. W. Li and M. Zhao, "On controllability of dependent siphons for deadlock prevention in generalized Petri nets," IEEE Transactions on Systems, Man, and Cybernetics, Part A, vol. 38, no. 2, pp. 369-384, 2008.

[6] M. Zhao and Y. Hou, "An iterative method for synthesizing non-blocking supervisors for a class of generalized petri nets using mathematical programming," Discrete Event Dynamic Systems, vol. 23, no. 1, pp. 3-26, 2011. 
[7] Z. W. Li, N. Q. Wu, and M. C. Zhou, "Deadlock control of automated manufacturing systems based on Petri nets - a literature review," IEEE Transactions on Systems, Man, and Cybernetics, Part C, vol. 42, no. 4, pp. 437-462, 2012.

[8] A. Nazeem and S. Reveliotis, "Maximally permissive deadlock avoidance for resource allocation systems with r/w-locks," Discrete Event Dynamic Systems, vol. 25, no. 1, pp. 31-63, 2015.

[9] M. Cabasino, A. Giua, and C. Seatzu., "Fault detection for discrete event systems using Petri nets with unobservable transitions," Automatica, vol. 46, no. 9, pp. 1531-1539, 2010.

[10] R. Cordone, A. Nazeem, L. Piroddi, and S. A. Reveliotis, "Designing optimal deadlock avoidance policies for sequential resource allocation systems through classification theory: Existence results and customized algorithms," IEEE Transactions on Automatic Control, vol. 58, no. 11, pp. 2772-2787, 2013.

[11] M. P. Cabasino, A. Giua, C. N. Hadjicostis, and C. Seatzu, "Fault model identification and synthesis in Petri nets," Discrete Event Dynamic Systems, vol. 25, no. 13, pp. 419-440, 2015.

[12] M. Pocci, I. Demongodin, N. Giambiasi, and A. Giua, "Synchronizing sequences on a class of unbounded systems using synchronized Petri nets," Discrete Event Dynamic Systems, vol. 26, no. 1, pp. 85-108, 2016.

[13] L. E. Holloway, B. H. Krogh, and A. Giua, "A survey of Petri net methods for controlled discrete event systems," Discrete Event Dynamic Systems: Theory and Applications, vol. 7, no. 2, pp. 151-190, 1997.

[14] J. Moody and P. Antsaklis, "Petri net supervisors for DES with uncontrollable and unobservable transitions," IEEE Transactions on Automatic Control, vol. 45, no. 3, pp. 462-476, 2000.

[15] M. V. Iordache and P. J. Antsaklis, "Supervision based on place invariants: A survey," Discrete Event Dynamic Systems, vol. 16, no. 4, pp. 4451-492, 2006.

[16] A. Giua, "Supervisory control of Petri nets with language specifications," in Control of discrete-event systems, C. Seatzu, M. Silva, and J. van Schuppen, Eds. London: Springer, 2013, vol. 433, pp. 235255.

[17] Z. Y. Ma, Z. W. Li, and A. Giua, "Design of optimal Petri net controllers for disjunctive generalized mutual exclusion constraints," IEEE Transactions on Automatic Control, vol. 60, pp. 1774-1785, 2015.

[18] Y. Ru, M. P. Cabasino, A. Giua, and C. N. Hadjicostis, "Supervisor synthesis for discrete event systems under partial observation and arbitrary forbidden state specifications," Discrete Event Dynamic Systems, vol. 24, no. 3, pp. 275-307, 2014. 
[19] A. Ghaffari, N. Rezg, and X. Xie, "Feedback control logic for forbidden — state problems of marked graphs: application to a real manufacturing system,' IEEE Transactions on Automatic Control, vol. 48, pp. 18-29, 2003.

[20] F. Basile, C. Carbone, and P. Chiacchio, "Feedback control logic for backward conflict free choice nets," IEEE Transactions on Automatic Control, vol. 52, no. 3, pp. 387-400, 2007.

[21] G. T. Murata, "Petri nets: properties, analysis and applications," Proceedings of the IEEE, vol. 77, no. 4, pp. 541-580, 1989.

[22] M. Uzam and M. C. Zhou, "An improved iterative synthesis method for liveness enforcing supervisors of flexible manufacturing systems," International Journal of Production Research, vol. 44, no. 10, pp. 1987-2030, 2006.

[23] _ _ "An iterative synthesis approach to Petri net based deadlock prevention policy for flexible manufacturing systems," IEEE Transactions on Systems, Man and Cybernetics, Part A, vol. 37, no. 3, pp. 362-371, 2007.

[24] Y. F. Chen and Z. W. Li, "Design of a maximally permissive liveness-enforcing supervisor with a compressed supervisory structure for flexible manufacturing systems," Automatica, vol. 47, no. 5, pp. 1028 1034, 2011.

[25] A. Giua, F. DiCesare, and M. Silva, "Generalized mutual exclusion constraints for Petri nets with uncontrollable transitions," in In Proceedings of the IEEE Int. Conf. on Systems, Man, and Cybernetics, Chicago, USA, 1992, pp. 947-949.

[26] L. E. Holloway, A. S. Khare, and Y. Gong, "Computing bounds for forbidden state reachability functions for controlled Petri nets," IEEE Transactions on Systems, Man, and Cybernetics, Part A, vol. 34, no. 2, pp. 219-228, 2004.

[27] J. L. Luo, W. M. Wu, H. Y. Su, and J. Chu, "Supervisor synthesis for enforcing a class of generalized mutual exclusion constraints on Petri nets," IEEE Transactions on Systems, Man, and Cybernetics, Part A, vol. 39, no. 6, pp. 1237-1246, 2009.

[28] J. L. Luo and K. Nonami, "Approach for transforming linear constraints on Petri nets," IEEE Transactions on Automatic Control, vol. 56, no. 12, pp. 2751-2765, 2011.

[29] S. G. Wang, C. Y. Wang, and M. C. Zhou, "A transformation algorithm for optimal admissible generalized mutual exclusion constraints on Petri nets with uncontrollable transitions," in In Proceedings of the IEEE Int. Conf. on Robotics and Automation, Shanghai, China, 2011, pp. 3745-3750. 
[30] F. Basile, R. Cordone, and L. Piroddi, "Integrated design of optimal supervisors for the enforcement of static and behavioral specifications in Petri net models," Automatica, vol. 49, no. 11, pp. 3432-3439, 2013.

[31] _ _ "A branch and bound approach for the design of decentralized supervisors in Petri net models," Automatica, vol. 52, pp. 322-333, 2015.

[32] J. H. Ye, Z. W. Li, and A. Giua, "Decentralized supervision of Petri nets with a coordinator," IEEE Transactions on Systems, Man, and Cybernetics: Systems, vol. 45, pp. 955-966, 2015.

[33] M. V. Iordache, P. Wu, F. Zhu, and P. J. Antsaklis, "Efficient design of Petri-net supervisors with disjunctive specifications," in In Proceedings of the IEEE Int. Conf. on Automation Science and Engineering, Madison, USA, 2013, pp. 936-941.

[34] F. Basile, P. Chiacchio, and A. Giua, "Suboptimal supervisory control of Petri nets in presence of uncontrollable transitions via monitor places," Automatica, vol. 42, no. 6, pp. 995-1004, 2006.

[35] J. Campos, C. Seatzu, and X. Xie, Formal Methods in Manufacturing, ser. Industrial Information Technology. CRC Press, 2014.

[36] J. L. Luo, W. M. Wu, H. Y. Su, and J. Chu, "Supervisor synthesis for enforcing GMECs on a controlled Petri net," in In Proceedings of the 25th American Control Conference, Minneapolis, USA, 2006, pp. $4165-4170$.

[37] — _ "Supervisor synthesis for a class of forbidden state problems in Petri nets," in In Proceedings of the 25th American Control Conference, Minneapolis, USA, 2006, pp. 4171-4176.

[38] L. E. Dickson, "Finiteness of the odd perfect and primitive abundant numbers with $n$ distinct prime factors," American Journal of Mathematics, vol. 35, no. 4, pp. 413-422, 1913.

[39] Z. Y. Ma, Z. W. Li, and A. Giua, "A constraint transformation technique for Petri nets with certain uncontrollable structures," in In Proceedings of the 12th International Workshop on Discrete Event Systems, Cachan, France, 2014, pp. 66-72. 